\title{
Serum IgG-induced microglial activation enhances neuronal cytolysis via the NO/ sGC/PKG pathway in children with opsoclonus-myoclonus syndrome and neuroblastoma
}

Xu Ding ${ }^{1 *+}$, Wei Yang ${ }^{2 \dagger}$, Qinghua Ren ${ }^{2}$, Jiajian Hu², Shen Yang ${ }^{2}$, Wei Han ${ }^{2}$, Jing Wang ${ }^{3}, X$ U Wang ${ }^{4}$ and Huanmin Wang ${ }^{2^{*}}$ (D)

\begin{abstract}
Background: Opsoclonus-myoclonus syndrome (OMS) is a rare neurological disease. Some children with OMS also have neuroblastoma (NB). We and others have previously documented that serum IgG from children with OMS and NB induces neuronal cytolysis and activates several signaling pathways. However, the mechanisms underlying OMS remain unclear. Here, we investigated whether nitric oxide (NO) from activated microglias and its cascade contribute to neuronal cytolysis in pediatric OMS.

Methods: The activation of cultured cerebral cortical and cerebellar microglias incubated with sera or lgG isolated from sera of children with OMS and NB was measured by the expression of the activation marker, cytokines, and NO. Neuronal cytolysis was determined after exposing to IgG-treated microglia-conditioned media. Using inhibitors and activators, the effects of NO synthesis and its intracellular cascade, namely soluble guanylyl cyclase (sGC) and protein kinase $G(P K G)$, on neuronal cytolysis were evaluated.

(Continued on next page)
\end{abstract}

\footnotetext{
* Correspondence: dingxu@bjmu.edu.cn; wanghuanmin2018@126.com

${ }^{+}$Xu Ding and Wei Yang contributed equally to this work.

'Laboratory of Nutrition and Development, Beijing Pediatric Research

Institute, Beijing Children's Hospital, Capital Medical University, National

Center for Children's Health, No. 56 Nan-li-shi Road, Xi-Cheng District, Beijing

100045, China

2Department of Surgical Oncology, Beijing Children's Hospital, Capital

Medical University, National Center for Children's Health, Beijing 100045,

China

Full list of author information is available at the end of the article
}

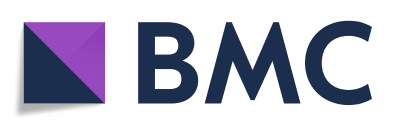

( ) The Author(s). 2020 Open Access This article is licensed under a Creative Commons Attribution 4.0 International License, which permits use, sharing, adaptation, distribution and reproduction in any medium or format, as long as you give appropriate credit to the original author(s) and the source, provide a link to the Creative Commons licence, and indicate if changes were made. The images or other third party material in this article are included in the article's Creative Commons licence, unless indicated otherwise in a credit line to the material. If material is not included in the article's Creative Commons licence and your intended use is not permitted by statutory regulation or exceeds the permitted use, you will need to obtain permission directly from the copyright holder. To view a copy of this licence, visit http://creativecommons.org/licenses/by/4.0/. The Creative Commons Public Domain Dedication waiver (http://creativecommons.org/publicdomain/zero/1.0/) applies to the data made available in this article, unless otherwise stated in a credit line to the data. 
(Continued from previous page)

Results: Incubation with sera or IgG from children with OMS and NB increased the activation of cerebral cortical and cerebellar microglias, but not the activation of astrocytes or the cytolysis of glial cells. Moreover, the cytolysis of neurons was elevated by conditioned media from microglias incubated with lgG from children with OMS and NB. Furthermore, the expression of NO, sGC, and PKG was increased. Neuronal cytolysis was relieved by the inhibitors of NO signaling, while neuronal cytolysis was exacerbated by the activators of NO signaling but not proinflammatory cytokines. The cytolysis of neurons was suppressed by pretreatment with the microglial inhibitor minocycline, a clinically tested drug. Finally, increased microglial activation did not depend on the Fab fragment of serum lgG.

Conclusions: Serum IgG from children with OMS and NB potentiates microglial activation, which induces neuronal cytolysis through the NO/sGC/PKG pathway, suggesting an applicability of microglial inhibitor as a therapeutic candidate.

Keywords: Opsoclonus-myoclonus syndrome, Neuroblastoma, Children, Microglial activation, Nitric oxide (NO), Soluble guanylyl cyclase (sGC), Protein kinase G (PKG)

\section{Background}

Opsoclonus-myoclonus syndrome (OMS) is a rare but devastating neurological disease, characterized by opsoclonus, myoclonus, and ataxia. Most patients suffer from persistent deficits in cognition, neurology, and behavior. Some children with OMS also have neuroblastoma (NB), although varied percentages have been reported [1-5]. Previously, we have documented that the insulin-like growth factor 1 (IGF-1)/phosphoinositide 3-kinase (PI3K) cascade is compensatively activated to alleviate neuronal cytolysis induced by serum IgG from children with OMS and NB [6], and others reported that the phosphorylation of extracellular signal-regulated kinase contributes to neuronal cytolysis in pediatric OMS [7]. Yet, the cellular and molecular mechanisms associated with neuronal cytolysis underlying pediatric OMS remain unclear.

Microglias are major immune effectors in the central nervous system (CNS) and have an important physiological function in inflammation [8]. Notably, patients with pediatric OMS exhibit increased expression of a microglial marker and proinflammatory cytokines in cerebrospinal fluid (CSF) and some children with OMS are post-infectious [9-11]. Furthermore, serum IgG or autoantibody existed in patients enhances microglial activation in Parkinson disease (PD) [12], amyotrophic lateral sclerosis (ALS) [13], and systemic lupus erythematosus (SLE) [14-16]. Thus, it is reasonable to hypothesize that serum IgG from children with OMS and NB may impact the activation of microglia.

Activated microglias release various neurotoxic molecules $[8,17]$, causing the loss of neurons in neurodegenerative diseases [18-20]. We and others have revealed the cytolysis of cultured cerebellar and cerebral neurons by sera or IgG from patients with pediatric OMS and NB [6, 7]. Additionally, activated microglias can synthesize and release nitric oxide (NO), which can activate soluble guanylyl cyclase (sGC) and protein kinase G (PKG) in neurons, thereby contributing to neuronal death by mitochondrial dysfunction [21, 22] and increased neuronal susceptibility to mitochondrial dysfunction [23]. However, whether the cytolysis of neurons can be induced by activated microglias in children with OMS and $\mathrm{NB}$ via the $\mathrm{NO} / \mathrm{sGC} / \mathrm{PKG}$ cascade is still unknown.

Here, we found that sera or serum IgG from children with OMS and NB induces the activation of cultured cerebral cortical and cerebellar microglias, but not astrocytes. The cytolysis of neurons is exerted by conditioned media from microglias treated with IgG from children with OMS and NB. Furthermore, the NO/sGC/PKG pathway contributes to neuronal cytolysis induced by conditioned media, and neuronal cytolysis can be almost completely suppressed by pretreatment with the microglial inhibitor minocycline, a clinically tested drug. Finally, increased microglial activation may depend on the Fc fragment of serum IgG rather than the Fab fragment. Our results suggest that serum IgG from children with OMS and NB increases the activation of cultured microglias, leading to the upregulation of NO, which subsequently activates sGC and PKG in neurons, thereby inducing neuronal cytolysis. These results also suggest that the microglial inhibitor, such as minocycline, may serve as a plausible therapeutic candidate.

\section{Methods \\ Subjects \\ Enrolled participants}

This project was reviewed and approved by the Ethics Committees of Beijing Children's Hospital, Capital Medical University (No. IEC-C-028-A10-V.05). Parents of participants signed written informed consent. Children were enrolled between January 2015 and December 2019 from Beijing Children's Hospital using internationally accepted diagnostic criteria for OMS [24] and they were Han Chinese. Ten children with OMS and NB were collected (OMS + NB). Control groups were 20 children with NB without OMS (NB), 10 age- and sex-matched 
healthy children (healthy control), 10 children with juvenile idiopathic arthritis (JIA) to reveal whether the effects on microglias are common to all IgG-related diseases, and 6 children with anti- $N$-methyl- $D$-aspartate receptor (NMDAR) encephalitis to investigate whether the effects on microglias are common to all autoantibody-mediated disorders of the CNS. Two individuals in the OMS + NB group, 3 individuals in the NB group, and all the individuals in the anti-NMDAR encephalitis were newly collected, while the rest subjects were previously described in our another study [6].

\section{Clinical information}

Demographic and clinical information is shown in Table 1. The age and gender of 5 groups, as well as the tumor stage of the NB and OMS + NB groups, were not statistically different. The age of subjects was at the time of our study, and thus, it may be older than the age of onset. Although a higher female sex ratio of approximately 1.2 was already evident in toddlers with OMS [2-4], only 10 children with OMS and NB in our study may not enough to reflect the gender difference, and other literatures with a small sample size also showed much lower (2 females/3 males) [25] or higher ratios ( 9 females/2males, $11 \mathrm{fe-}$ males/4 males) [7, 26]. Moreover, the age and gender of subjects may be affected by whether timely visiting to hospitals, misdiagnosis as other diseases before, genetic background, or willingness to enroll in the study. All the children in the JIA group had systemiconset JIA. Electroencephalogram (EEG) results of children with OMS were clinically normal, while EEG results of all the children with anti-NMDAR encephalitis were abnormal. All the blood samples of the NB and OMS + NB groups were recruited before surgery for $\mathrm{NB}$, and most of the blood samples were recruited before any treatment. After collection, sera were stored at $-80{ }^{\circ} \mathrm{C}$.

According to the criteria for evaluating OMS [1], the degree of ataxia, opsoclonus, ataxia/gait, ataxia/stance, and $\mathrm{mood} / \mathrm{sleep}$ disturbance of patients with OMS was graded from 0 to 3 and summarized into OMS score in Table 2. The frequency of symptoms was similar to a previous report [4].

\section{Purification of lgG and preparation of the Fab fragment}

As we previously used [6], $100 \mu \mathrm{l}$ of protein G agaroses (Thermo Fisher Scientific, Sunnyvale, CA, USA) were applied to sera. The IgG-free fraction of sera in supernatants was collected after centrifugation at $10000 \mathrm{~g}$ for 10 min. The IgG fraction of sera was eluted by $0.1 \mathrm{M}$ glycine- $\mathrm{HCl}(\mathrm{pH} 2.7)$ after washing with $0.01 \mathrm{M}$ phosphate buffer saline (PBS), and then neutralization buffer was added. The BCA assay (Pierce, Rockford, IL, USA) was applied to determine IgG concentration.

The Fab fragment of IgG was prepared by enzymatic digestion [16]. Pepsin (Sigma-Aldrich, St. Louis, MO, USA) was mixed with IgG at a ratio of $1: 20$ and then incubated at $37^{\circ} \mathrm{C}$ for $6 \mathrm{~h}$. The $\mathrm{pH}$ of solution was adjusted to 7.4 to stop the digestion. HiTrap Protein G HP columns (GE Healthcare, Freiburg, Germany) and Amicon Ultra-15 centrifugal filters (Merck Millipore, Billerica, MA, USA) were used to obtain the Fab fragment.

\section{Primary cultures of microglias or astrocytes separately}

Primary cultures of microglias [27] or astrocytes [28] were prepared as previously described. Sprague Dawley rats at postnatal day 1 were used, which were provided by the Department of Experimental Animal Sciences, Capital Medical University (Beijing, China). The cerebral cortex and cerebellum were cut and digested in $0.1 \%$ trypsin at $37^{\circ} \mathrm{C}$ for $20 \mathrm{~min}$, and then, tissues were

Table 1 Demographic and clinical data

\begin{tabular}{|c|c|c|c|c|c|}
\hline & $n$ & $\begin{array}{l}\text { Age enrolled in our study } \\
\text { (month) }\end{array}$ & $\begin{array}{l}\text { Gender (female/ } \\
\text { male) }\end{array}$ & $\begin{array}{l}\text { Tumor } \\
\text { stage }\end{array}$ & Treatment before serum collection \\
\hline Healthy children & 10 & $41.9 \pm 1.7$ & $5 / 5$ & - & - \\
\hline NB & 20 & $44.5 \pm 6.4$ & $9 / 11$ & $\begin{array}{l}\text { I: } 4 \\
\text { II: } 9 \\
\text { III: } 6 \\
\text { IV: } 1\end{array}$ & $\begin{array}{l}13 \text { children: no treatment } \\
7 \text { children: chemotherapy, receiving vincristine, } \\
\text { cyclophosphamide, adriamycin, etoposide, } \\
\text { and cisplatin }\end{array}$ \\
\hline $\mathrm{OMS}+\mathrm{NB}$ & 10 & $35.6 \pm 9.9$ & $5 / 5$ & $\begin{array}{l}\text { I: } 3 \\
\text { II: } 4 \\
\text { III: } 3 \\
\text { IV: } 0\end{array}$ & $\begin{array}{l}8 \text { children: no treatment } \\
1 \text { child: steroid } \\
1 \text { child: intravenous immune globulin }\end{array}$ \\
\hline $\begin{array}{l}\text { Juvenile idiopathic arthritis } \\
(J \mid A)\end{array}$ & 10 & $40.8 \pm 10.5$ & $6 / 4$ & - & $\begin{array}{l}6 \text { children: no treatment } \\
3 \text { children: nonsteroidal anti-inflammatory drugs } \\
1 \text { child: received steroid }\end{array}$ \\
\hline Anti-NDMAR encephalitis & 6 & $54.3 \pm 9.9$ & $4 / 2$ & - & $\begin{array}{l}2 \text { children: no treatment } \\
4 \text { children: steroid and intravenous immune } \\
\text { globulin }\end{array}$ \\
\hline
\end{tabular}


Table 2 OMS scores of children with OMS and NB at the time of the blood draw

\begin{tabular}{llllll}
\hline Symptom & \multicolumn{3}{l}{ Number of children } & $\%$ \\
\cline { 2 - 5 } & Grade 0 & Grade 1 & Grade 2 & Grade 3 & \\
\hline Opsoclonus & 1 & 4 & 1 & 4 & 90 \\
Myoclonus & 3 & 5 & 2 & 0 & 70 \\
Ataxia/gait & 4 & 4 & 2 & 0 & 60 \\
Ataxia/stance & 6 & 4 & 0 & 0 & 40 \\
Mood/sleep disturbance & 5 & 5 & 0 & 0 & 50 \\
\hline
\end{tabular}

triturated gently. After filtration through a $30-\mu \mathrm{m}$ cell drainer (BD Biosciences Discovery Labware, Bedford, MA, USA), cell suspensions were centrifuged at 1500 rpm for 5 min and resuspended in Dulbecco's modified Eagle's medium (Life Technologies, Rockville, MD, USA) with $10 \%$ inactivated low-endotoxin fetal bovine serum.

For primary culture of microglias, mixed glial cultures were shaken at $200 \mathrm{rpm}$ for $2 \mathrm{~h}$ after seeding onto lysinecoated dishes for 7 days. After pelleting, floating cells were subcultured at $3.0 \times 10^{5}$ cells $/ 2000 \mu \mathrm{l}$ medium/well in 6 -well plates or at $1.0 \times 10^{4}$ cells/200 $\mu \mathrm{lmedium/well}$ in 96-well plates. One day after subculture, the medium was fully replaced by macrophage serum-free medium. Cells were used 2 days later.

For primary culture of astrocytes, mixed glial cultures were shaken at $200 \mathrm{rpm}$ for $2 \mathrm{~h}$ after seeding onto dishes for 10 days. Adherent cells were trypsinized $(0.05 \%)$ and subcultured at $3.0 \times 10^{5}$ cells/2000 $\mu \mathrm{l}$ medium/well in 6well plates with glial culture medium. One day after subculture, plates were manually shaken for $1 \mathrm{~min}$ and the medium was fully replaced with glial culture medium. Cells were used 7-10 days later.

\section{Primary culture of neurons}

Primary culture of neurons was carried out according to methods previously described [6]. The cerebral cortex and cerebellum of Sprague Dawley rats (16 to 18 days old) were used. Notably, $24 \mathrm{~h}$ after seeding neurons, cytosine arabinoside at $10 \mu \mathrm{M}$ was added to suppress glial proliferation and prepare neuron-enriched cultures.

\section{Treatment of sera, lgG, or chemicals}

\section{Microglias or astrocytes incubated with sera or $\lg G$}

As we used previously [6], the culture medium of microglias or astrocytes was replaced with a fresh medium containing sera or IgG from children (6-well plates in a 2000- $\mu$ l volume: $40 \mu \mathrm{l}$ sera of children, 1:50 diluted; 100-200 $\mu \mathrm{g}$ IgG; $1000-1400 \mu \mathrm{g}$ the IgG-free fraction; 96well plates in a $200-\mu \mathrm{l}$ volume: $4 \mu \mathrm{l}$ sera of children, 1:50 diluted; 10-20 $\mu \mathrm{g}$ IgG). Microglias or astrocytes were incubated with sera or IgG for $48 \mathrm{~h}$ before performing further assays, such as ELISA, or getting conditioned media. To explore whether increased microglial activation is simply induced by higher concentration of IgG, commercially available human IgG (Sigma-Aldrich) was dissolved in normal saline and was added into each well with the medium at the final concentration of $0.1 \mu \mathrm{g} / \mu \mathrm{l}$ and the highest concentration in the OMS + NB group. Each serum or IgG of an individual parallelly treated 3 wells of glial cells. Cluster of differentiation $11 \mathrm{~b}(\mathrm{CD} 11 \mathrm{~b})$ is a marker of microglial activation. Microglias to examine the expression of $\mathrm{CD} 11 \mathrm{~b}$ and conditioned media to detect cytokines or neuronal cytolysis were got from the same well.

\section{Neurons incubated with conditioned media}

In order to avoid the direct effects of serum IgG on neurons [6], instead of co-culture of microglias and neurons and treating both kinds of cells with IgG at the same time, here we cultured microglias or neurons separately. IgG from children was added into the culture media of microglias to collect conditioned media, and then, conditioned media were filtered with protein $G$ agarose to get the IgG-free fraction. Finally, the culture media of neurons were replaced with IgG-free conditioned media from microglias. This method has been used before [29].

\section{Microglias or neurons treated with chemicals}

To investigate the role of $\mathrm{NO}$ and its intracellular cascade in neuronal cytolysis, the NO synthesis inhibitor 7nitroindazole (7-NINA) was added into the media of microglias $30 \mathrm{~min}$ before treatment with the IgG fraction, whereas the sGC inhibitor $1 \mathrm{H}-1,2,4$ oxadiazolo-4,3-a quinoxalin-1-one (ODQ) or the PKG inhibitor Rp-8BrPET-cGMP (the inhibitor of both PKG type I and type II) (all from Tocris Biosciences, Bristol, UK) was added into the culture media of neurons $30 \mathrm{~min}$ before the replacement of conditioned media from microglias. While 7NINA or Rp-8Br-PET-cGMP was dissolved in normal saline and added into the medium to make a final concentration of $10 \mu \mathrm{M}$ or $1 \mu \mathrm{M}$, ODQ was dissolved in $5 \%$ dimethyl sulfoxide (DMSO, Sigma-Aldrich) at a final concentration of $10 \mu \mathrm{M}$.

Moreover, pharmacological activators of the NO signaling pathway were also tested. The NO-donor $S$-nitroso- $N$ acetylpenicillamine (SNAP) was added into the media of microglias $30 \mathrm{~min}$ before treatment of IgG from children, while the sGC activator 3-5-hydroxymethyl-2-furyl-1-benzyl-indazole (YC-1) or the PKG activator 8Br-cGMP (the activator of both PKG type I and type II) (all from Tocris Biosciences) was added into the culture media of neurons $30 \mathrm{~min}$ before the replacement of IgG-treated microglia conditioned media. Whereas SNAP or YC-1 was dissolved in $5 \%$ DMSO and added into the medium at $100 \mu \mathrm{M}$ or $1 \mu \mathrm{M}, 8 \mathrm{Br}$-cGMP was dissolved in normal saline at a final concentration of $1 \mu \mathrm{M}$. The final concentration of DMSO was $0.01 \%$. Additionally, minocycline (Sigma-Aldrich), an 
inhibitor of microglial activation, was given 30 min before SNAP or IgG from children. Minocycline was dissolved in normal saline and added into the medium at $20 \mu \mathrm{M}$.

Recombinant IL-1 $\beta$, IL-6, TNF- $\alpha$, or MCP-1 (all from R \& D Systems, Minneapolis, MN, USA) was used 30 min before the replacement of neuronal medium. Cytokines were dissolved in $0.01 \mathrm{M}$ PBS containing $0.1 \%$ bovine serum albumin (BSA) and were added into the culture medium of neurons at $100 \mathrm{pg} / \mathrm{ml}, 100 \mathrm{pg} / \mathrm{ml}, 80$ $\mathrm{ng} / \mathrm{ml}$, and $20 \mathrm{ng} / \mathrm{ml}$, respectively. The concentrations of inhibitors, activators, and recombinant cytokines were selected based on previous studies [23, 30].

To investigate the role of IGF/PI3K signaling in neuronal cytolysis, recombinant IGF-1 (R \& D Systems) was added into the culture media of neurons $30 \mathrm{~min}$ before the replacement of conditioned media from microglias. IGF-1 was dissolved in $0.01 \mathrm{M}$ PBS and was added at the final concentration of $10 \mathrm{nM}$. To detect the effects of PI3K on neuronal cytolysis, the PI3K inhibitor LY294002 (Sigma-Aldrich) was added into the culture media of neurons $30 \mathrm{~min}$ before IGF-1. LY294002 was dissolved in $5 \%$ DMSO and was added at the final concentration of $20 \mu \mathrm{M}$.

\section{Enzyme-linked immunosorbent assay (ELISA)}

Cultured microglias, astrocytes, or neurons were lysed in RIPA buffer (KeyGen Biotechnology, Nanjing, China) after washing by PBS. Commercially available ELISA kits were applied to explore the expression of CD11b (Cloud-Clone, Houston, TX, USA) in microglias, glial fibrillary acidic protein (GFAP) (Abcam, Cambridge, MA, USA) in astrocytes, and PI3K (CUSABIO, Wuhan, China) [6] in neurons. The concentrations of CD11b, GFAP, and PI3K were normalized to the amount of total protein, which was detected at the same time as ELISA using the BCA protein assay kit.

Moreover, the culture medium of microglias was used to assess the concentrations of proinflammatory cytokines IL-1 $\beta$ (Bio-Swamp, Wuhan, China), IL-6 (Promocell, Heidelberg, Germany), TNF- $\alpha$ (Abcam), and MCP1 (Abcam) by commercially available ELISA kits following manufacturer's instructions.

\section{Nitrite assay}

To calculate the concentration of $\mathrm{NO}$ in the culture medium of microglias, nitrite was measured, which is a product resulting from the reaction of NO with molecular oxygen. Briefly, $50 \mu \mathrm{l}$ of cell culture media and an equal volume of Griess reagent $(0.1 \%$ naphtyletylenediamine dihydrochloride, $1 \%$ sulphanilamide, $2.5 \%$ phosphoric acid, Sigma-Aldrich) were added in 96-well plates. The absorbance at $550 \mathrm{~nm}$ was determined, and standard curves with sodium nitrite $\left(\mathrm{NaNO}_{2}\right.$, SigmaAldrich) were generated [27].

\section{Cytolysis assay}

Cytoplasmic lactate dehydrogenase (LDH), an indicator of plasma membrane-damaged cells, was measured by the cytotoxicity detection kit (Roche, Indianapolis, IN, USA) [6]. Cytolysis of neurons or microglias was detected using the same method. Briefly, $100 \mu \mathrm{l}$ of culture supernatants of neurons or microglias was mixed with an equal volume of reaction mixture followed by incubation for $30 \mathrm{~min}$. LDH was detected by the absorbance at $490 \mathrm{~nm}$. Maximum LDH release was induced by $1 \%$ Triton $\mathrm{X}-100$, while the background control was untreated neurons or microglias. The percentage of specific cytolysis was determined using the following formula: [(experimental value-background control)/(maximum valuebackground control)] $\times 100$.

\section{Western blot}

To assess the protein expression of CD11b in the cell membrane, the cell membrane fraction of microglias was isolated using the plasma membrane protein extraction kit (Abcam). Protein concentration was determined by the BCA protein assay kit. After gel electrophoresis, proteins were transferred to PVDF membranes. PVDF membranes with the cell membrane fraction were rinsed briefly in distilled water and stained with Ponceau S solution (Po-S) $(0.5[\mathrm{w} / \mathrm{v}]$ in $1 \%[\mathrm{v} / \mathrm{v}]$ acetic acid $)$ for $2 \mathrm{~min}$, rinsed in distilled water to remove excess stain, and imaged. Protein levels were normalized to Po-S as a loading control (100$140 \mathrm{kDa}$ ) [31]. After blocking, rabbit anti-CD11b antibody (127 kDa, 1:1000, ab133357, Abcam) was incubated at $4{ }^{\circ} \mathrm{C}$ overnight. Following washing, PVDF membranes were then incubated for $1 \mathrm{~h}$ at room temperature with HRPconjugated secondary antibody. Finally, an enzymatic chemiluminescence kit (Thermo Fisher Scientific) was used to visualize protein bands, and the intensity of protein bands was quantified using Quantity One software (Bio-Rad, Hercules, CA, USA).

To assess the protein expression of $\mathrm{sGC}$ or PKG in whole-cell lysates, neurons were homogenized in RIPA buffer. PVDF membranes with whole-cell lysates were blocked before incubating with rabbit anti-sGC $\beta 1$ subunit antibody (70 kDa, 1:1000, G4405, Sigma-Aldrich), rabbit anti-PKG type I antibody (78 kDa, 1:1000, 3248, Cell Signaling Technology, Danvers, MA, USA), rabbit anti-PKG type II antibody ( $87 \mathrm{kDa}, 1: 1000$, SAB4502387, SigmaAldrich), or mouse anti-glyceraldehyde 3-phosphate dehydrogenase (GAPDH) antibody (40 kDa, 1:5000, ab8245, Abcam). GAPDH was used as a loading control. Other steps were the same as described above.

\section{Statistical analyses}

All the data were expressed as mean \pm SEM. Statistical analyses were carried out using GraphPad Prism version 6.0 software for Windows (GraphPad Software Inc., San 
Diego, CA, USA). One-way analysis of variance (ANOVA) followed by Dunnett's multiple comparison test was used for multiple analyzation. The statistically significant level was considered at $p<0.05$.

\section{Results}

The activation of cerebral cortical and cerebellar microglias is increased by sera or IgG from children with OMS and NB

Besides the cerebellum, emerging evidence has shown that the cerebral cortex has structural and functional changes in OMS patients. First, most OMS patients have neurological handicaps in cerebral functions, such as deficits in attention, memory, and language [4, 32]. Second, brain imaging of OMS patients shows changes in the cerebrum. Cerebral cortical thickness is reduced across the motor and visual areas in patients with pediatric OMS [33]. A patient with OMS reveals significant nodular enhancing lesions at gray-white junction of bilateral cerebral hemispheres by magnetic resonance imaging [34]. Another patient shows decreased metabolism in the bilateral occipital lobes and increased functional connectivity, including the primary- and motionsensitive visual cortex [35]. Therefore, both cerebral cortical and cerebellar microglias were exposed to sera or the IgG fraction from children with OMS and NB.

The expression of CD11b, a marker of microglial activation, was upregulated in cerebral cortical microglias incubated with sera from children with OMS and NB (3.41 \pm $0.32 \mathrm{ng} / \mathrm{mg}$ total protein OMS + NB, $0.99 \pm 0.09 \mathrm{ng} / \mathrm{mg}$ total protein NB, $1.09 \pm 0.12 \mathrm{ng} / \mathrm{mg}$ total protein healthy control, $p<0.001$ vs NB, $p<0.001$ vs healthy control), whereas $\mathrm{CD} 11 \mathrm{~b}$ concentration was not statistically changed by sera of children with only NB at least under our experimental conditions (Fig. 1a). Moreover, IgG isolated from sera upregulated CD11b expression from $0.91 \pm$ $0.10 \mathrm{ng} / \mathrm{mg}$ total protein in the NB group and $0.88 \pm 0.07$ $\mathrm{ng} / \mathrm{mg}$ total protein in the healthy control group to $2.95 \pm$ $0.23 \mathrm{ng} / \mathrm{mg}$ total protein in the OMS $+\mathrm{NB}$ group $(p<$ 0.001 vs NB, $p<0.001$ vs healthy control, Fig. 1c). With respect to cerebellar microglias, the concentration of CD11b was also increased after incubation with sera or IgG from the OMS + NB group compared with those from the NB or healthy control group (Fig. 1b, d). However, neither commercially available human IgG nor IgG from children with JIA or anti-NMDAR encephalitis had a significant impact on the concentration of CD11b (Fig. 1c, d), suggesting that upregulation of $\mathrm{CD} 11 \mathrm{~b}$ induced by serum IgG from children with OMS and NB is not simply induced by increased dose of IgG, is not common to all diseases with IgG, and is not common to all autoantibodymediated disorders of the CNS. In addition, no alteration of $\mathrm{CD} 11 \mathrm{~b}$ concentration was observed after treatment with the IgG-free fraction (Fig. 1e, f), which suggested that the upregulation of CD11b induced by sera mainly depends on the IgG fraction. Consistently, the protein expression of $\mathrm{CD} 11 \mathrm{~b}$ in the cell membrane fraction was increased in the cerebral cortical and cerebellar microglias incubated with OMS + NB IgG (Fig. 1 g, h).

To further investigate the effects of serum IgG from children with OMS and NB on microglial activation, the concentrations of proinflammatory cytokines (including IL-1 $\beta$, IL- 6 , TNF- $\alpha$, and MCP-1) and NO in the media of cerebral cortical and cerebellar microglias were detected by ELISA. The releases of IL- $1 \beta$, IL- 6 , TNF- $\alpha$, and MCP-1 from cerebral cortical microglias exposed to OMS $+\mathrm{NB}$ sera were elevated (IL-1 $\beta$ : $105.4 \pm 10.48 \mathrm{pg} / \mathrm{ml}$ OMS + $\mathrm{NB}, 43.99 \pm 4.29 \mathrm{pg} / \mathrm{ml} \mathrm{NB}, 39.83 \pm 5.64 \mathrm{pg} / \mathrm{ml}$ healthy control, $p<0.001$ vs NB, $p<0.001$ vs healthy control; IL6: $107.8 \pm 11.20 \mathrm{pg} / \mathrm{ml}$ OMS + NB, $61.61 \pm 3.67 \mathrm{pg} / \mathrm{ml}$ $\mathrm{NB}, 49.11 \pm 5.53 \mathrm{pg} / \mathrm{ml}$ healthy control, $p<0.01 \mathrm{vs} \mathrm{NB}, p$ $<0.001$ vs healthy control; TNF- $\alpha$ : $169.1 \pm 14.32 \mathrm{pg} / \mathrm{ml}$ $\mathrm{OMS}+\mathrm{NB}, 100.3 \pm 9.15 \mathrm{pg} / \mathrm{ml} \mathrm{NB}, 90.24 \pm 6.76 \mathrm{pg} / \mathrm{ml}$ healthy control, $p<0.001$ vs NB, $p<0.001$ vs healthy control; MCP-1: $294.1 \pm 9.30 \mathrm{pg} / \mathrm{ml} \mathrm{OMS} \mathrm{+} \mathrm{NB,} 181.7 \pm 7.93$ $\mathrm{pg} / \mathrm{ml} \mathrm{NB}, 184.7 \pm 9.59 \mathrm{pg} / \mathrm{ml}$ healthy control, $p<0.001$ vs NB, $p<0.001$ vs healthy control), whereas the incubation with NB sera had no such effect (Fig. 2a1). Furthermore, the levels of IL- $1 \beta$, IL- 6 , TNF- $\alpha$, and MCP-1 secreted from cerebral cortical microglias were increased to $109.6 \pm 12.09 \mathrm{pg} / \mathrm{ml}, 133.2 \pm 11.28 \mathrm{pg} / \mathrm{ml}, 225.3 \pm 9.84$ $\mathrm{pg} / \mathrm{ml}$, and $324.2 \pm 6.68 \mathrm{pg} / \mathrm{ml}$ after incubation with OMS + NB IgG ( $p<0.001$ vs NB, $p<0.001$ vs healthy control, Fig. 2b1). In cerebellar microglias, similar impacts of sera and IgG were observed (Fig. 2c1, d1). Consistent with the results of cytokines, $\mathrm{NO}$ expression was also improved in the media of cerebral cortical microglias (Fig. 2a2, b2) and cerebellar microglias (Fig. 2c2, d2) treated with OMS + NB sera or IgG. Together with the expression of CD11b, these results suggested that the activation of cultured cerebral cortical and cerebellar microglias was upregulated by serum IgG from children with OMS and NB.

In order to explore whether serum IgG-enhanced activation is specific to microglias or common to glial cells in the CNS, we detected the activation of cerebral cortical and cerebellar astrocytes. Using the marker of astrocytic activation, we found that the expression of GFAP was not significantly changed in cerebral cortical astrocytes (Fig. 3a, c) and cerebellar astrocytes (Fig. 3b, d) incubated with sera or IgG from the OMS $+\mathrm{NB}$ group compared with those from the $\mathrm{NB}$ group and healthy control group at least under our experimental conditions, suggesting that the enhancement of activation induced by IgG from children with OMS and NB is specific to microglias, but not astrocytes.

Previously, we have revealed that serum IgG from children with OMS and NB induces cytolysis in cultured neurons [6], whether serum IgG impacts the cytolysis of microglias or 


\section{Cerebral cortical microglia Cerebellar microglia}
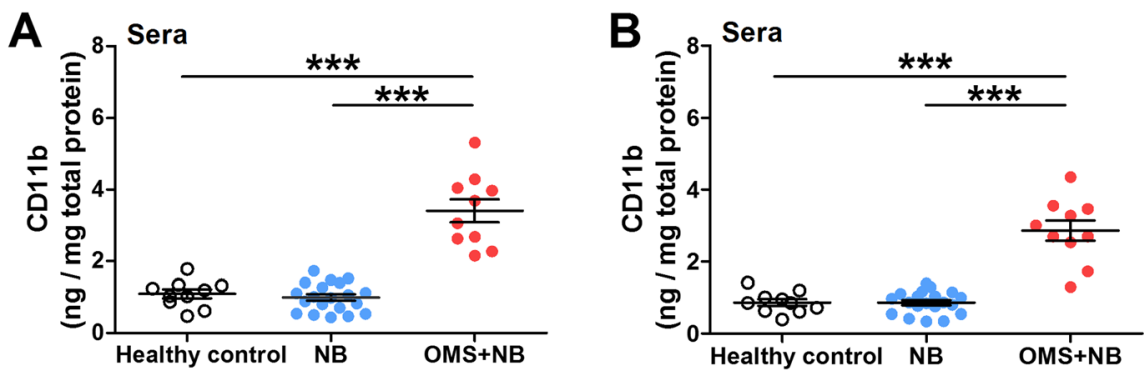

\begin{tabular}{|llll|}
\hline$\circ$ Healthy control & $\bullet$ NB & $\bullet$ OMS + NB \\
$\bullet \operatorname{lgG}$ & $\bullet$ JIA & $\bullet$ anti-NMDAR encephalitis \\
\hline
\end{tabular}
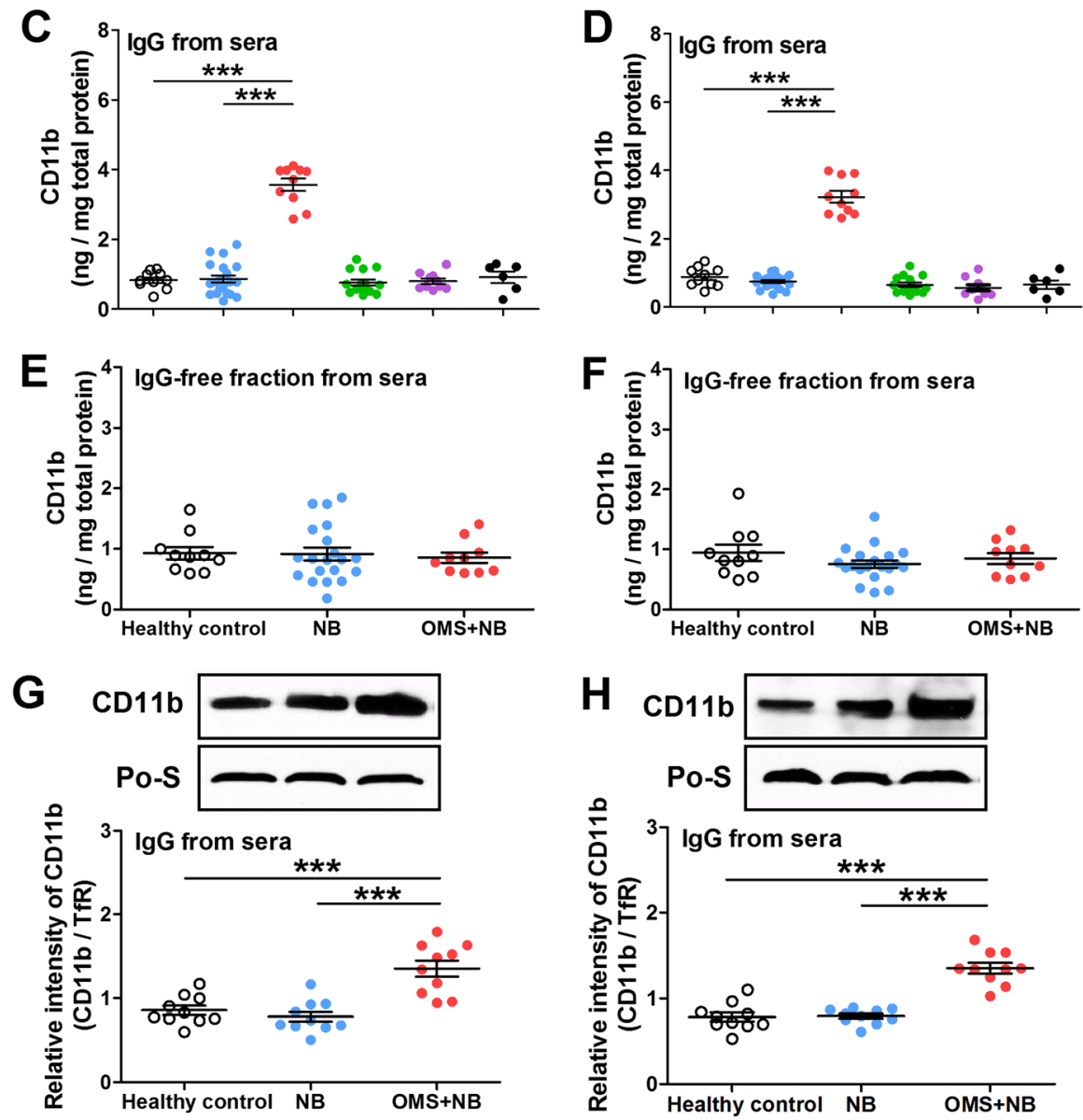

Fig. 1 CD11b expression in cerebral cortical and cerebellar microglias incubated with OMS + NB sera or lgG. Note that the concentration of CD11b, a marker of microglial activation, was upregulated in cerebral cortical microglias (a) and cerebellar microglias (b) incubated with sera from children with OMS and NB (OMS + NB) compared with those from children with NB or healthy children. The lgG fraction (c, $\mathbf{d})$ of the OMS + NB group also elevated the level of CD1 1b, but not the IgG-free fraction (e, f). No alteration was observed in the expression of CD1 1b after treatment with commercially available human lgG, the lgG fraction from children with juvenile idiopathic arthritis (JIA) or anti-NMDAR encephalitis (c, d). The expression of CD11b in the cell membrane of microglias was upregulated by $\operatorname{lgG}$ of the OMS + NB group $(\mathbf{g}, \mathbf{h}) .{ }^{* * *} p<0.001$, one-way ANOVA, $n=10$ (health control), $n=20$ (NB), $n=10$ (OMS + NB), $n=15$ (lgG), $n=10$ (JIA), $n=6$ (anti-NMDAR encephalitis). $n=10 /$ group (g, $\mathbf{h}$, Western blot)

astrocytes needs further study. We observed that neither the cytolysis of cerebral cortical microglias (Fig. 4a, c) and cerebellar microglias (Fig. 4b, d) treated with OMS + NB sera or
IgG, nor the cytolysis of cerebral cortical astrocytes (Fig. 4e, g) and cerebellar astrocytes (Fig. 4f, h) treated with OMS + NB sera or IgG was statistically changed, which suggested 


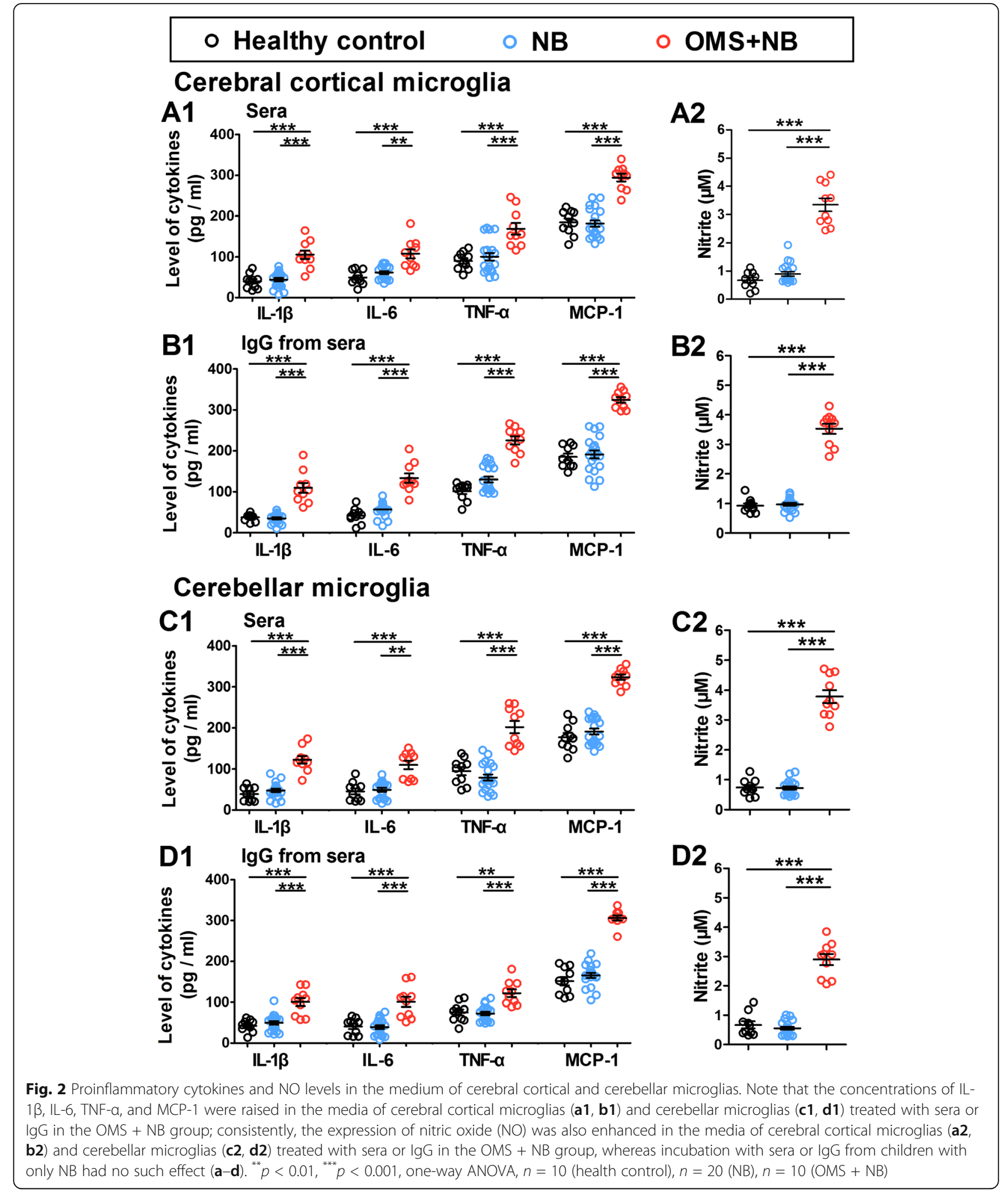

that preincubation with sera or IgG from children with OMS and NB specially upregulates microglial activation rather than the cytolysis of microglias and astrocytes at least under our experimental conditions.
The cytolysis of neurons is induced by conditioned media from microglias treated with OMS + NB IgG

It has been established that activated microglias contribute to neuron death by secreting various neurotoxic 


\section{Cerebral cortical astrocyte Cerebellar astrocyte}
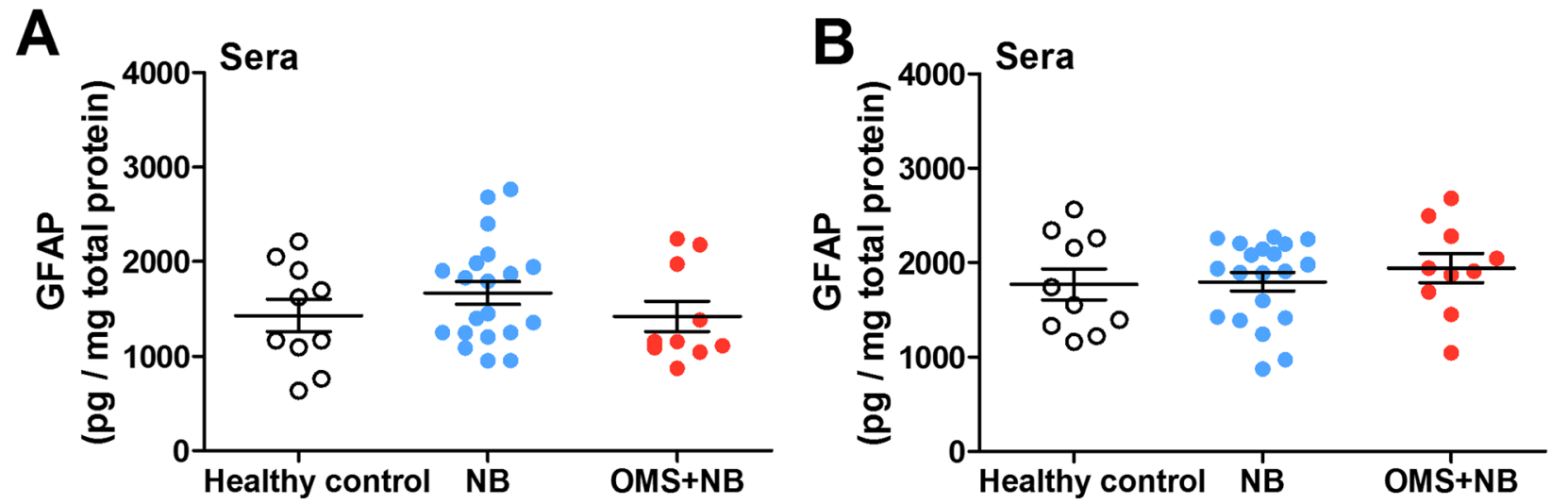

C
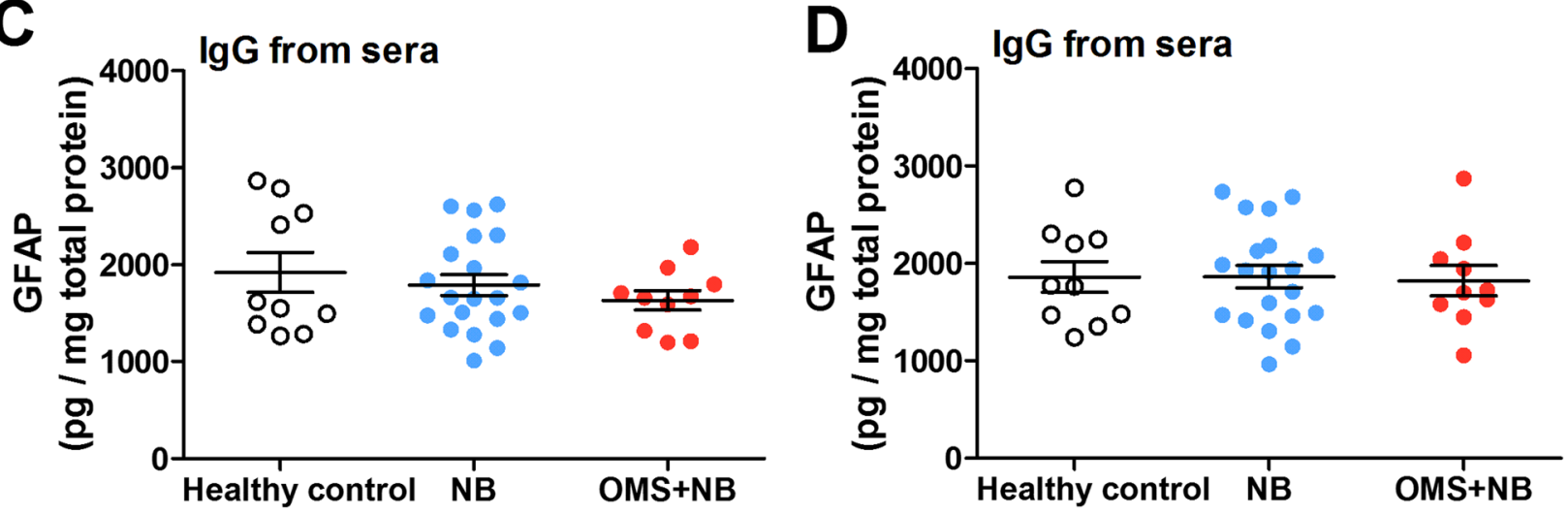

Fig. 3 GFAP concentration in cerebral cortical and cerebellar astrocytes incubated with OMS + NB sera or lgG. Note that the expression of GFAP, a marker of astrocytic activation, was not significantly changed in cerebral cortical and cerebellar astrocytes incubated with sera $(\mathbf{a}, \mathbf{b})$ or lgG (c, d) from children with OMS and NB compared with those from NB patients and healthy control at least under our experimental design. $p>0.05$, one-way ANOVA, $n=10$ (health control), $n=20$ (NB), and $n=10$ (OMS $+\mathrm{NB}$ )

molecules in multiple disorders $[8,17]$ and serum IgG from patients with OMS and NB enhances neuronal death $[6,7,36]$, we therefore explored whether neuronal cytolysis is enhanced by microglial activation in OMS. After getting rid of the remaining IgG, serum IgGtreated microglia conditioned media were collected and were replaced the culture media of neurons. As expected, incubation with conditioned media from cerebral cortical and cerebellar microglias increased the cytolysis of neurons in the same brain regions (cerebral cortical neuron: $31.95 \pm 1.09 \%$ OMS + NB, $13.45 \pm 1.12 \% \mathrm{NB}$, $14.53 \pm 1.08 \%$ healthy control; cerebellar neuron: 31.21 $\pm 1.25 \% \mathrm{OMS}+\mathrm{NB}, 10.50 \pm 1.27 \% \mathrm{NB}, 12.75 \pm 1.34 \%$ healthy control; $p<0.001$ vs NB, $p<0.001$ vs healthy control, Fig. 5a, b). Moreover, conditioned media from cerebral cortical microglias to cerebellar neurons or the exchanged situation had similar results (cerebral cortical neuron: $25.67 \pm 2.71 \%$ OMS + NB, $9.00 \pm 1.21 \% \mathrm{NB}$, $9.85 \pm 1.95 \%$ healthy control; cerebellar neuron: $27.24 \pm$ $2.55 \%$ OMS $+\mathrm{NB}, 11.79 \pm 1.06 \% \mathrm{NB}, 10.03 \pm 2.86 \%$ healthy control; $p<0.001$ vs NB, $p<0.001$ vs healthy control, Fig. 5c, d). Conversely, we observed no alteration in the cytolysis of neurons treated with conditional media from astrocytes (Fig. 5e, f). Taken together, these results suggested that conditioned media from cerebral cortical or cerebellar microglias rather than astrocytes induce the cytolysis of neurons.

$\mathrm{NO} / \mathrm{sGC} / \mathrm{PKG}$ signaling contributes to conditioned mediainduced neuronal cytolysis

To investigate the mechanisms of cytolysis induced by conditioned media from microglias treated with OMS + $\mathrm{NB}$ IgG, we focus on the NO/sGC/PKG pathway, for its role in neuronal death is well documented [21-23] and the production of $\mathrm{NO}$ from microglias was raised in the $\mathrm{OMS}+\mathrm{NB}$ group (Fig. 2a2-d2). We first examined the alterations of sGC and PKG abundance. Enzymatic activity of sGC needs its $\beta$ subunit, and although two types of $\beta$ subunit ( $\beta 1$ and $\beta 2$ ) have been cloned, only $\beta 1$ subunit has been shown to exist at the protein level in the brain 


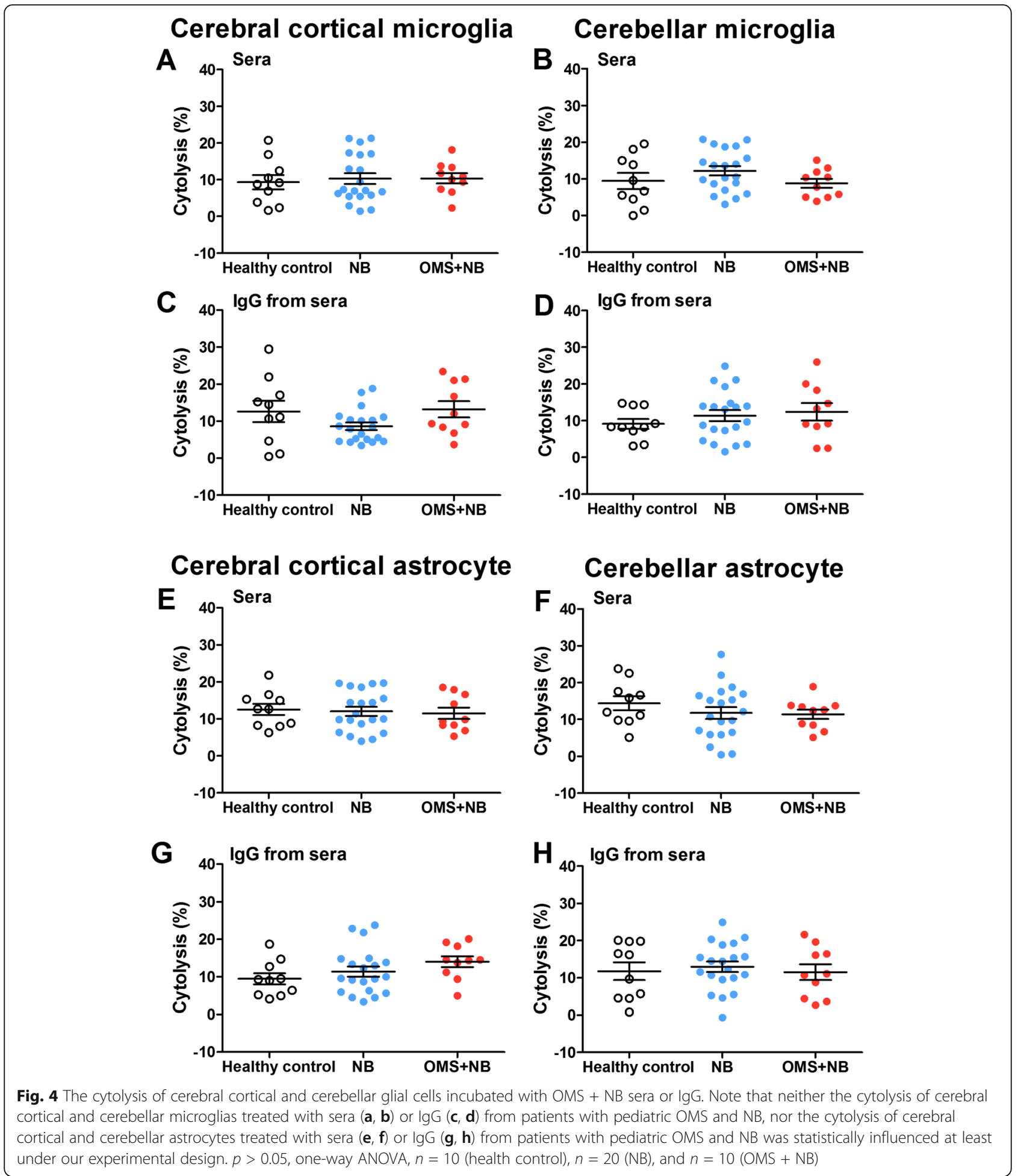

[37]. Two types of PKG (PKG I and PKG II) have been identified in mammalian tissues, both are related to cell apoptosis, proliferation [38, 39], and brain function [40, 41]. Our results showed that the protein expression of $\beta 1$ subunit of sGC was upregulated in cerebral cortical and cerebellar neurons after incubation with conditioned media of the OMS + NB group (cerebral cortical neuron: $1.54 \pm 0.08$ OMS $+\mathrm{NB}, 0.76 \pm 0.03 \mathrm{NB}, 0.72 \pm 0.06$ healthy control, $p<0.001$ vs NB, $p<0.001$ vs healthy control; cerebellar neuron: $1.56 \pm 0.10 \mathrm{OMS}+\mathrm{NB}, 0.76$ $\pm 0.05 \mathrm{pg} / \mathrm{ml} \mathrm{NB}, 0.77 \pm 0.06 \mathrm{pg} / \mathrm{ml}$ healthy control, $p<$ 0.001 vs $\mathrm{NB}, p<0.001$ vs healthy control; Fig. $6 \mathrm{a}, \mathrm{b})$, 


\section{Cerebral cortical neuron}

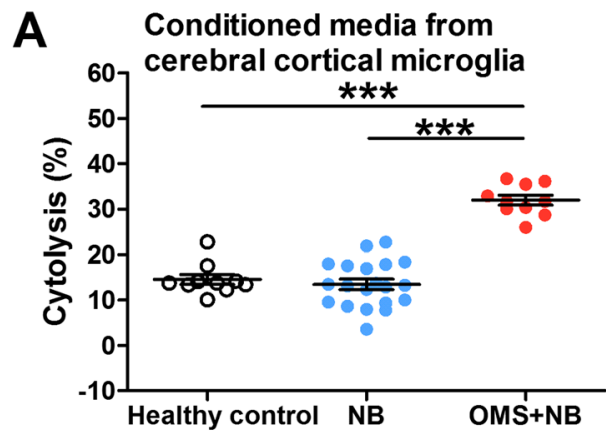

C

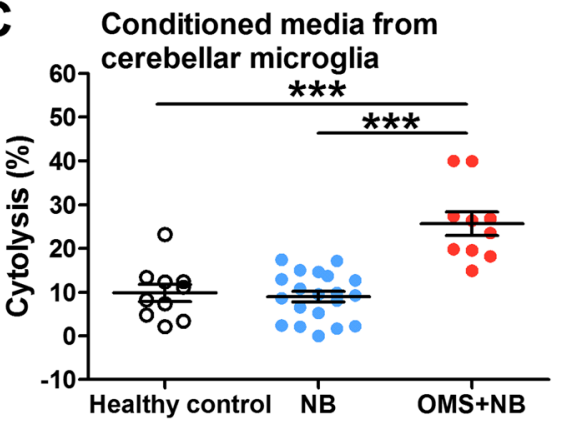

E

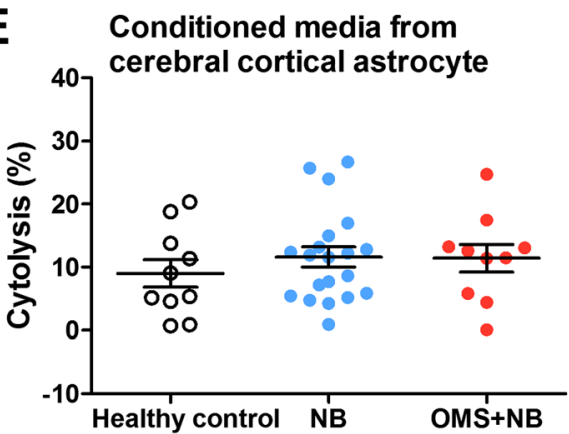

\section{Cerebellar neuron}
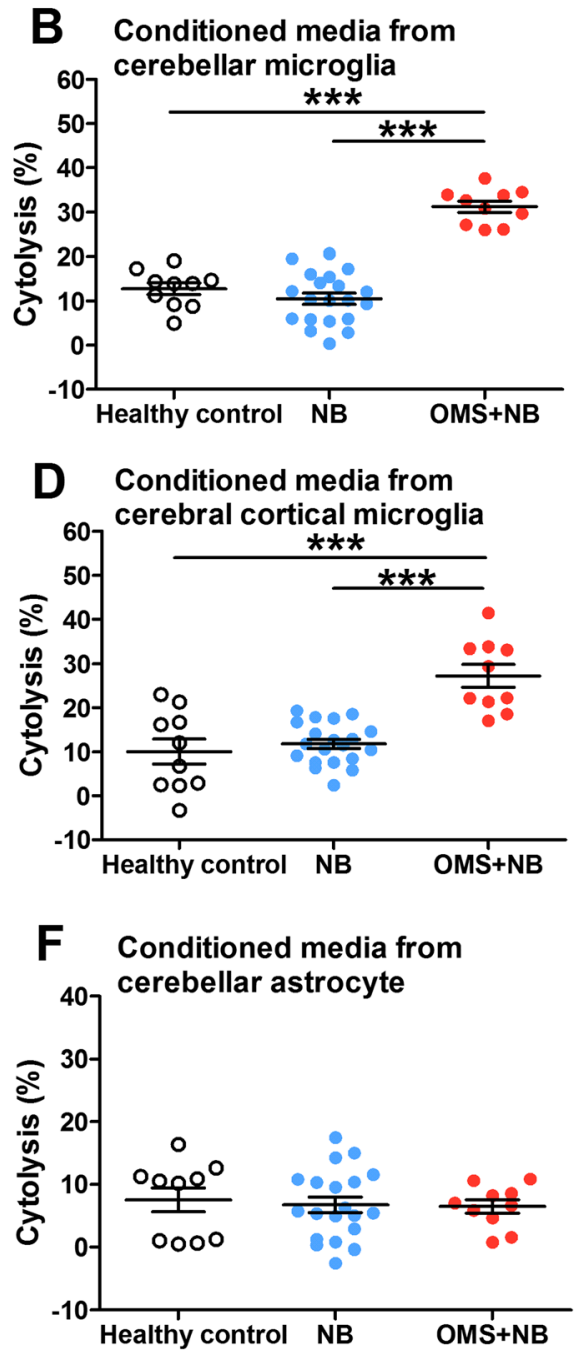

Fig. 5 Neuronal cytolysis induced by conditioned media from cerebral cortical and cerebellar microglias. After microglias incubated with OMS + $\mathrm{NB} \operatorname{lgG}$, conditioned media were collected, got rid of the remaining lgG, and replaced the culture media of neurons. Note that preincubation with conditioned media from cerebral cortical and cerebellar microglias enhanced the cytolysis of neurons in the same brain regions (a, b) or exchanged brain regions $(\mathbf{c}, \mathbf{d})$, while no alteration was observed in the cytolysis of neurons incubated with conditional media from astrocytes (e, f). ${ }^{* * *} p<0.001$, one-way ANOVA, $n=10$ (health control), $n=20$ (NB), and $n=10$ (OMS + NB)

and the expression of PKG I and PKG II was also increased (Fig. 6c-f).

Next, the effects of inhibitors of NO synthesis and NO-activated intracellular pathway were assessed. Pretreatment with the NO synthesis inhibitor 7-NINA to microglias before OMS + NB IgG alleviated the cytolysis of cerebral cortical and cerebellar neurons (cerebral cortical neuron: $6.50 \pm 1.46 \% 7$-NINA, OMS + NB vs 26.91 $\pm 1.45 \%$ saline, OMS $+\mathrm{NB}, p<0.001 ; 26.91 \pm 1.45 \%$ saline, OMS + NB vs $8.36 \pm 1.10 \%$ saline, NB, $p<0.001$; cerebellar neuron: $11.30 \pm 3.14 \%$ 7-NINA, OMS + NB vs $28.72 \pm 2.43 \%$ saline, OMS + NB, $p<0.001 ; 28.72 \pm$ $2.43 \%$ saline, OMS + NB vs $11.50 \pm 1.83 \%$ saline, $\mathrm{NB}, p$ $<0.001$; Fig. 7a, b). Similarly, pretreatment with the sGC inhibitor ODQ or the PKG inhibitor Rp-8Br-PET-cGMP ameliorated the cytolysis of cerebral cortical and cerebellar neurons (Fig. 7c-f).

Furthermore, the effects of activators of NO synthesis and $\mathrm{NO}$-activated intracellular pathway were examined. The cytolysis of cerebral cortical neurons was exaggerated after incubation with conditioned media from microglias pretreated with the NO-donor SNAP $30 \mathrm{~min}$ before OMS + NB IgG $(45.05 \pm 1.74 \%$ SNAP, OMS + $\mathrm{NB}$ vs $26.09 \pm 2.08 \%$ DMSO, OMS + NB, $p<0.001$; $26.09 \pm 2.08 \%$ DMSO, OMS + NB vs $7.12 \pm 0.98 \%$ DMSO, NB, $p<0.001$; Fig. 8a), and the cytolysis of cerebellar neurons was exacerbated by treatment with SNAP before OMS + NB IgG $(51.51 \pm 2.55 \%$ SNAP, OMS + 


\section{Cerebral cortical neuron}

A
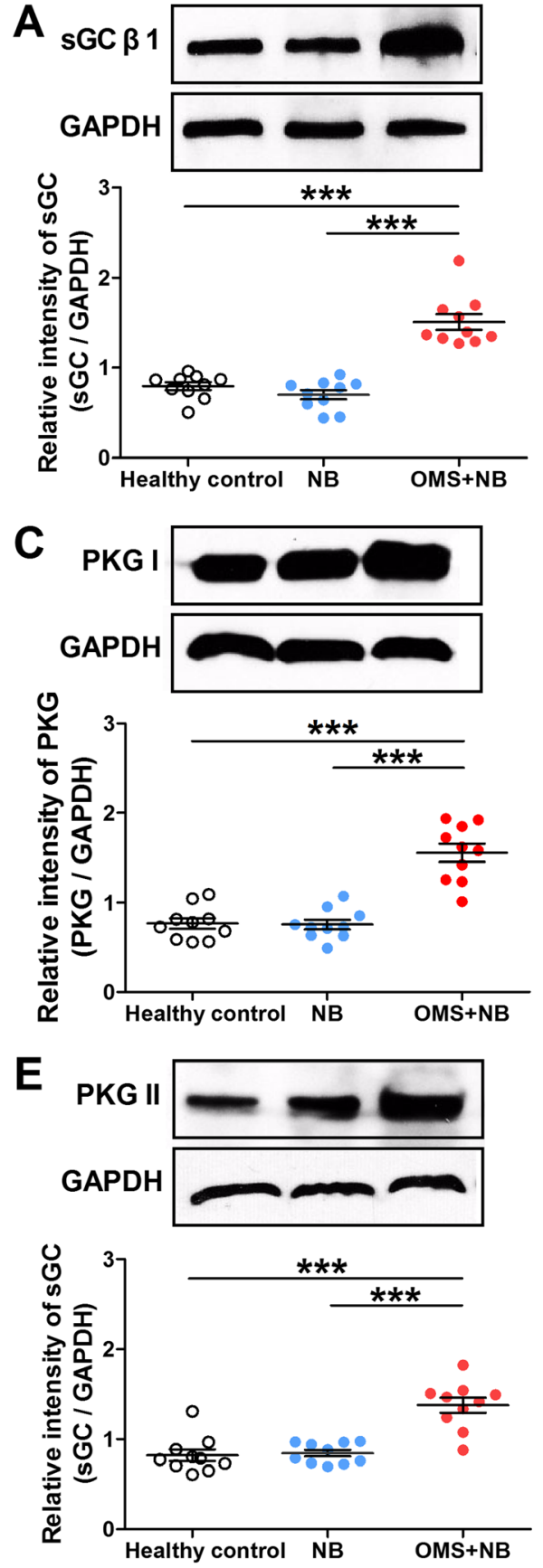

\section{Cerebellar neuron}
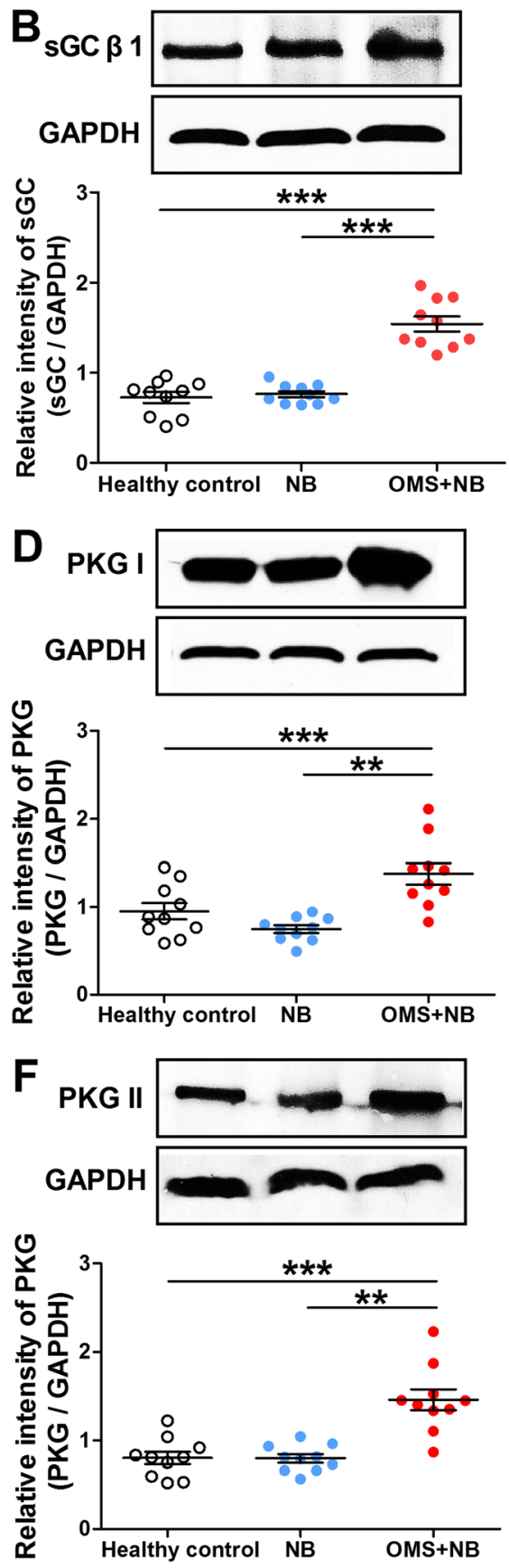

Fig. 6 The expression of sGC and PKG in cultured neurons incubated with conditioned media. Note that the protein expression of $\beta 1$ subunit of sGC $(\mathbf{a}, \mathbf{b})$, PKG I (c, d), and PKG II (e, $\mathbf{f})$ was increased in cerebral cortical and cerebellar neurons incubated with conditioned media from microglias treated with OMS + NB IgG. ${ }^{* *} p<0.01,{ }^{* * *} p<0.001$, one-way ANOVA, $n=10 /$ group

$\mathrm{NB}$ vs $35.27 \pm 1.87 \% \mathrm{DMSO}$, OMS $+\mathrm{NB}, p<0.001$ $35.27 \pm 1.87 \%$ DMSO, OMS $+\mathrm{NB}$ vs $8.06 \pm 1.21 \%$ DMSO, NB, $p<0.001$; Fig. 8b). Pretreatment with the activator of $\mathrm{sGC}$ or PKG, namely $\mathrm{YC}-1$ or $8 \mathrm{Br}-\mathrm{cGMP}$, also exacerbated cerebral cortical and cerebellar neuronal cytolysis induced by conditioned media from microglias stimulated with OMS + NB IgG (Fig. 8c-f). In addition, pretreatment with minocycline, an inhibitor of microglia, almost completely blocked the exacerbatory effects of these abovementioned activators and neuronal cytolysis induced by IgG-treated microglia conditioned media (Fig. 8a-f). Collectively, these results suggested 


\section{Conditioned media from cerebral cortical microglia to neuron \\ Conditioned media from cerebellar microglia to neuron}

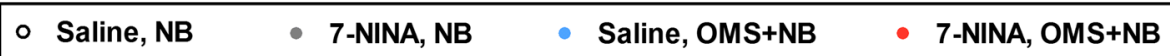
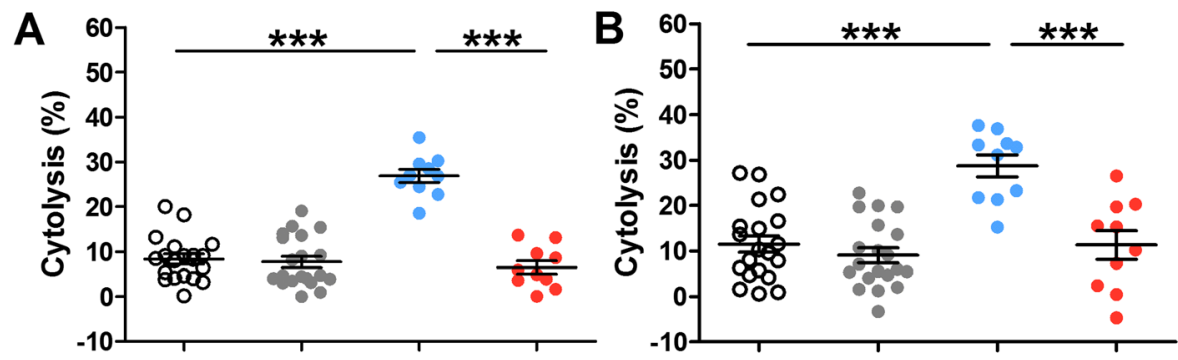

\begin{tabular}{|c|c|c|c|c|}
\hline DMSO, NB & - ODQ, NB & & DMSO, OMS+NB & - ODQ, OMS+NB \\
\hline
\end{tabular}
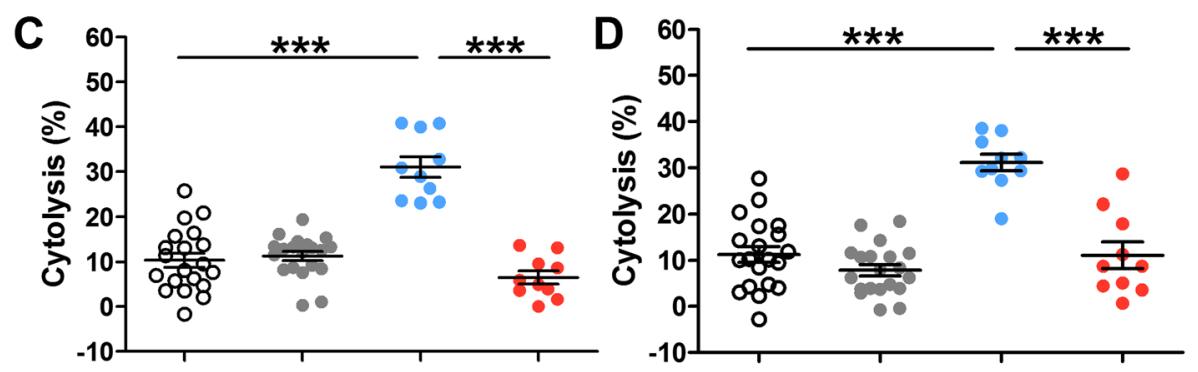
- Saline, NB
- Rp-8Br-PET-cGMP, NB
Saline, OMS+NB
- Rp-8Br-PET-cGMP, OMS+NB
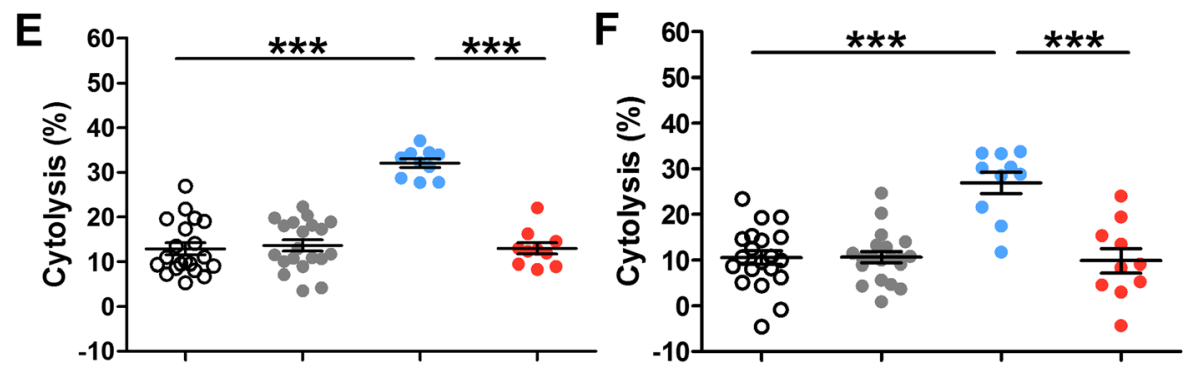

Fig. 7 The alleviation of neuronal cytolysis by pretreatment with pharmacological inhibitors of the NO cascade. Note that the cytolysis of cerebral cortical neurons (a) and cerebellar neurons (b) induced by conditioned media from microglias treated with OMS + NB lgG was relieved by the NO synthesis inhibitor 7-NINA, the cytolysis of cerebral cortical neurons (c) and cerebellar neurons (d) was reduced by the sGC inhibitor ODQ, and the cytolysis of cerebral cortical neurons (e) and cerebellar neurons (f) was abrogated by Rp-8Br-PET-cGMP, an inhibitor of PKG. ${ }^{* * *} p<0.001$, one-way ANOVA, $n=20$ (vehicle, NB; inhibitor, NB), $n=10$ (vehicle, OMS + NB; inhibitor, OMS + NB)

that the $\mathrm{NO} / \mathrm{sGC} / \mathrm{PKG}$ cascade plays a vital role in neuronal cytolysis induced by conditioned media from microglias treated with serum IgG from children with OMS and $\mathrm{NB}$, which depends on the activation of microglias.

The expression of proinflammatory cytokines in the culture medium of microglias incubated with OMS + NB IgG (Fig. 2a1-d1) and in CSF of OMS children [10] were upregulated, which raises the possibility that cytokines may have similar effects on neuronal cytolysis. Unexpectedly, our results showed that the cytolysis of cerebral cortical and cerebellar neurons induced by conditioned media from microglias preincubated with OMS + NB IgG was not influenced by IL-1 $\beta$ (cerebral cortical neuron: $32.89 \pm 1.70 \%$ IL-1 $\beta$, OMS + NB vs $33.23 \pm$ $1.37 \%$ 0.1\% BSA, OMS + NB, $p>0.05 ; 33.23 \pm 1.37 \%$ $0.1 \%$ BSA, OMS + NB vs $10.65 \pm 1.76 \% 0.1 \%$ BSA, NB, $p$ $<0.001$; cerebellar neuron: $27.55 \pm 2.32 \%$ IL-1 $\beta$, OMS + $\mathrm{NB}$ vs $31.26 \pm 1.88 \% 0.1 \%$ BSA, OMS + NB, $p>0.05$; $31.26 \pm 1.88 \% 0.1 \%$ BSA, OMS + NB vs $11.48 \pm 1.39 \%$ $0.1 \%$ BSA, NB, $p<0.001$; Fig. 9a, b). Also, enhanced 


\section{Conditioned media from Conditioned media from cerebral cortical microglia cerebellar microglia to neuron to neuron}

\begin{tabular}{|ll|l|}
\hline$\circ$ DMSO, NB $\quad$ SNAP, NB & DMSO, OMS+NB SNAP, OMS+NB \\
\hline Saline, SNAP, OMS+NB & - Minocycline, SNAP, OMS+NB \\
\hline
\end{tabular}
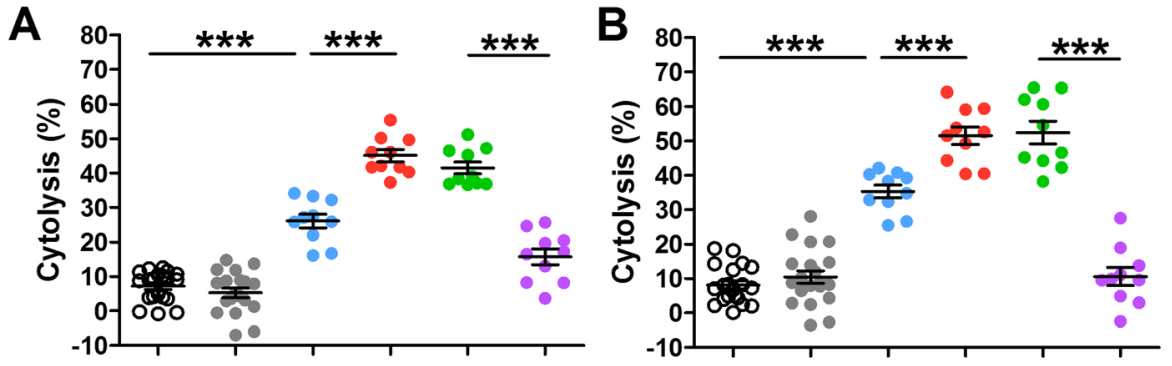

\begin{tabular}{|c|c|c|}
\hline$\circ$ & $\begin{array}{l}\text { DMSO, NB } \bullet \mathrm{YC}-1, \mathrm{NB} \\
\text { Saline, YC-1, OMS+NB }\end{array}$ & $\begin{array}{l}\text { DMSO, OMS+NB YC-1, OMS+NB } \\
\text { Minocycline, YC-1, OMS+NB }\end{array}$ \\
\hline
\end{tabular}
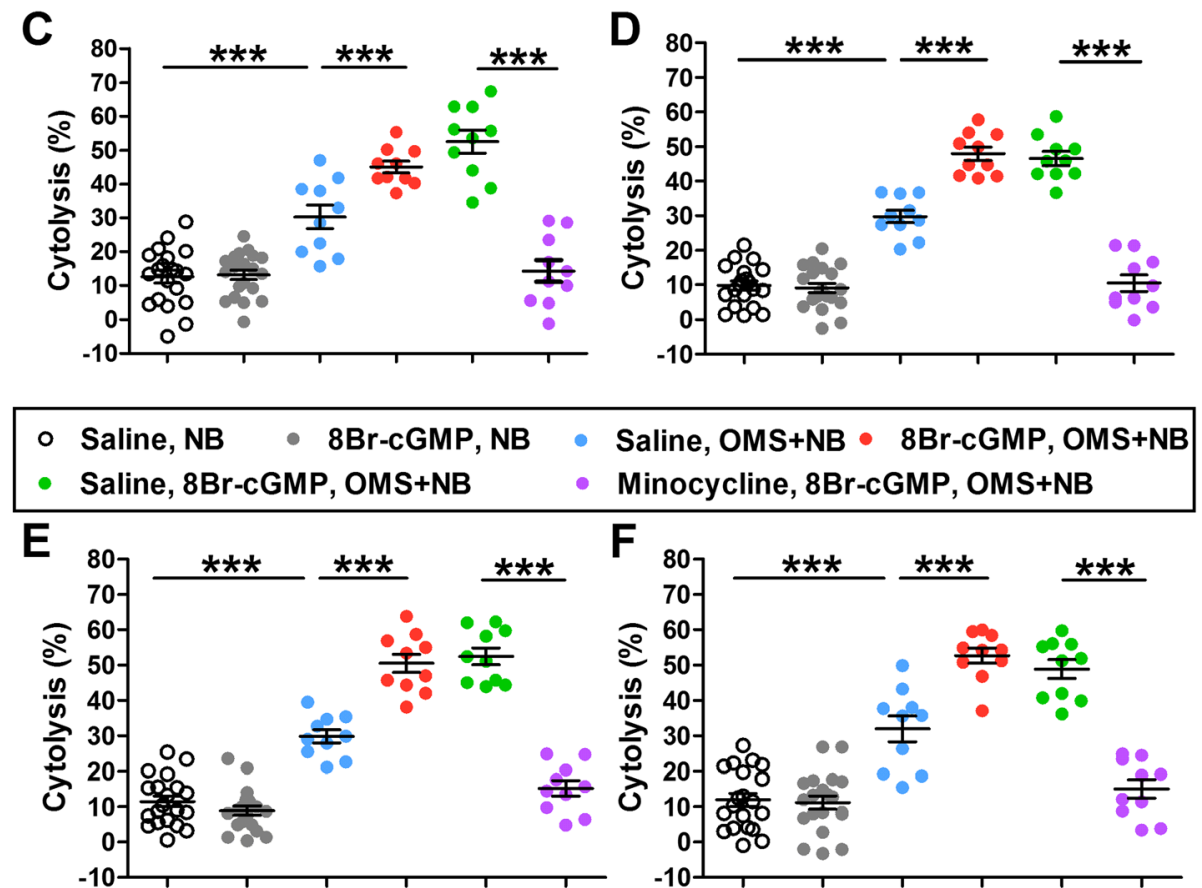

Fig. 8 The exaggeration of neuronal cytolysis by pretreatment with pharmacological activators of the NO pathway. Note that the cytolysis of cerebral cortical neurons (a) and cerebellar neurons (b) induced by conditioned media from microglias treated with OMS + NB IgG was exacerbated by the NO-donor SNAP, and the cytolysis of cerebral cortical neurons (c) and cerebellar neurons (d) was also upregulated by YC-1, an activator of sGC. Similarly, the PKG activator 8BrcGMP had exaggerated effects on the cytolysis of cerebral cortical neurons (e) and cerebellar neurons (f). The exaggerated effects of SNAP, YC-1, or 8Br-cGMP and neuronal cytolysis induced by conditioned media were suppressed by pretreatment with minocycline, an inhibitor of microglias $(\mathbf{a}-\mathbf{f}) .{ }^{* * *} p<0.001$, one-way ANOVA, $n=20$ (vehicle, NB; activator, NB), $n=10$ (vehicle, OMS + NB; activator, OMS + NB; saline, activator, OMS + NB; minocycline, activator, OMS + NB)

cytolysis of cerebral cortical and cerebellar neurons was not affected by IL-6, TNF- $\alpha$, or MCP-1 (Fig. 9c-h). These results suggested that the application of cytokines does not have similar effects of $\mathrm{NO}$ on the cytolysis of neurons induced by IgG-treated microglia conditioned media.
The activation of cerebral cortical and cerebellar microglias may depend on the Fc fragment of serum IgG rather than the Fab fragment

IgG contains the Fab fragment and the Fc fragment, and the Fab fragment combines with targeted antigens, while the Fc fragment interacts with $\mathrm{Fc} \gamma$ receptor $(\mathrm{Fc} \gamma \mathrm{R})$. Fc $\gamma \mathrm{R}$ 


\section{Conditioned media from cerebral cortical microglia to neuron \\ Conditioned media from cerebellar microglia to neuron}

\begin{tabular}{|lllllll}
\hline$\circ$ & $0.1 \%$ BSA, NB $\bullet \mathrm{IL}-1 \beta, \mathrm{NB}$ & $\bullet 0.1 \%$ BSA, OMS+NB $\bullet \mathrm{IL}-1 \beta$, OMS+NB \\
\hline
\end{tabular}
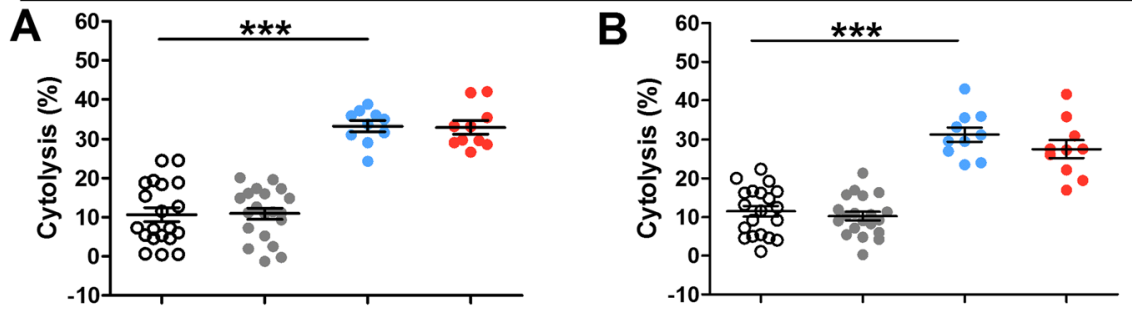

\begin{tabular}{|lllll|}
\hline$\circ$ & $0.1 \%$ BSA, NB & IL-6, NB & $-0.1 \%$ BSA, OMS+NB $\bullet$ IL-6, OMS+NB \\
\hline
\end{tabular}
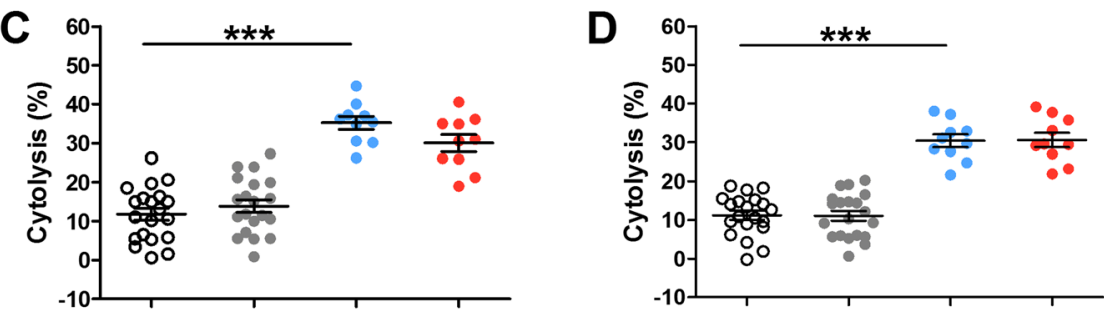

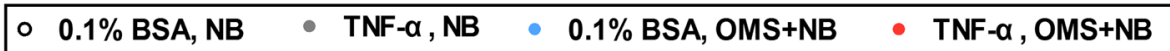
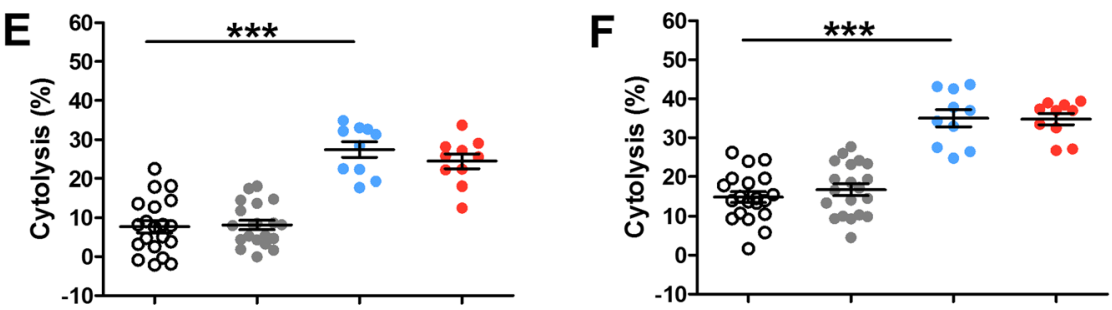

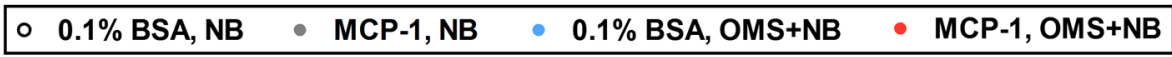
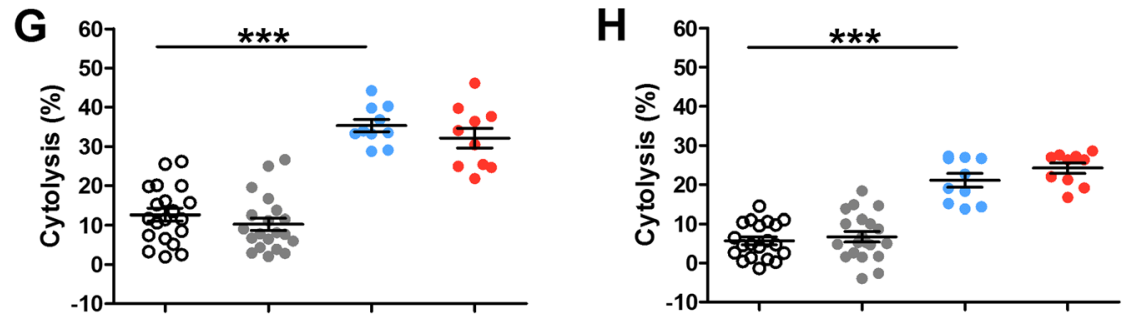

Fig. 9 The effects of cytokines on neuronal cytolysis induced by conditioned media from microglias. Note that the cytolysis of cerebral cortical neurons (a) and cerebellar neurons (b) induced by conditioned media from microglias preincubated with OMS + NB IgG was not influenced by IL-1 $\beta$, and the cytolysis of cerebral cortical neurons (c) and cerebellar neurons (d) was also not changed by IL-6. Consistently, the cytolysis of cerebral cortical neurons (e) and cerebellar neurons (f) was not affected by TNF-a, and the cytolysis of cerebral cortical neurons (g) and cerebellar neurons (h) was not changed by MCP-1. $p>0.05 ; 0.1 \%$ BSA, OMS + NB vs cytokine, OMS + NB. ${ }^{* * *} p<0.001 ; 0.1 \%$ BSA, NB vs cytokine, NB. Oneway ANOVA, $n=20$ (0.1\% BSA, NB; cytokine, NB), $n=10$ (0.1\% BSA, OMS + NB; cytokine, OMS + NB)

is expressed on the surface of microglias and other immune effector cells and mediates immune reactions in the brain. In order to explore whether increased microglial activation depends on the Fab fragment or the Fc fragment of serum IgG, we detected the effects of the Fab fragment on the expression of CD11b in microglias and the concentration of $\mathrm{NO}$ in the culture medium of microglias. The results showed that the expression of CD11b was not significantly changed in cerebral cortical and cerebellar microglias incubated with the Fab fragment from the OMS + NB group compared with that from the NB group and healthy control group (Fig. 10a, b). Consistently, the release of NO was not significantly changed in cerebral cortical and cerebellar microglias 


\section{Cerebral cortical microglia Cerebellar microglia}
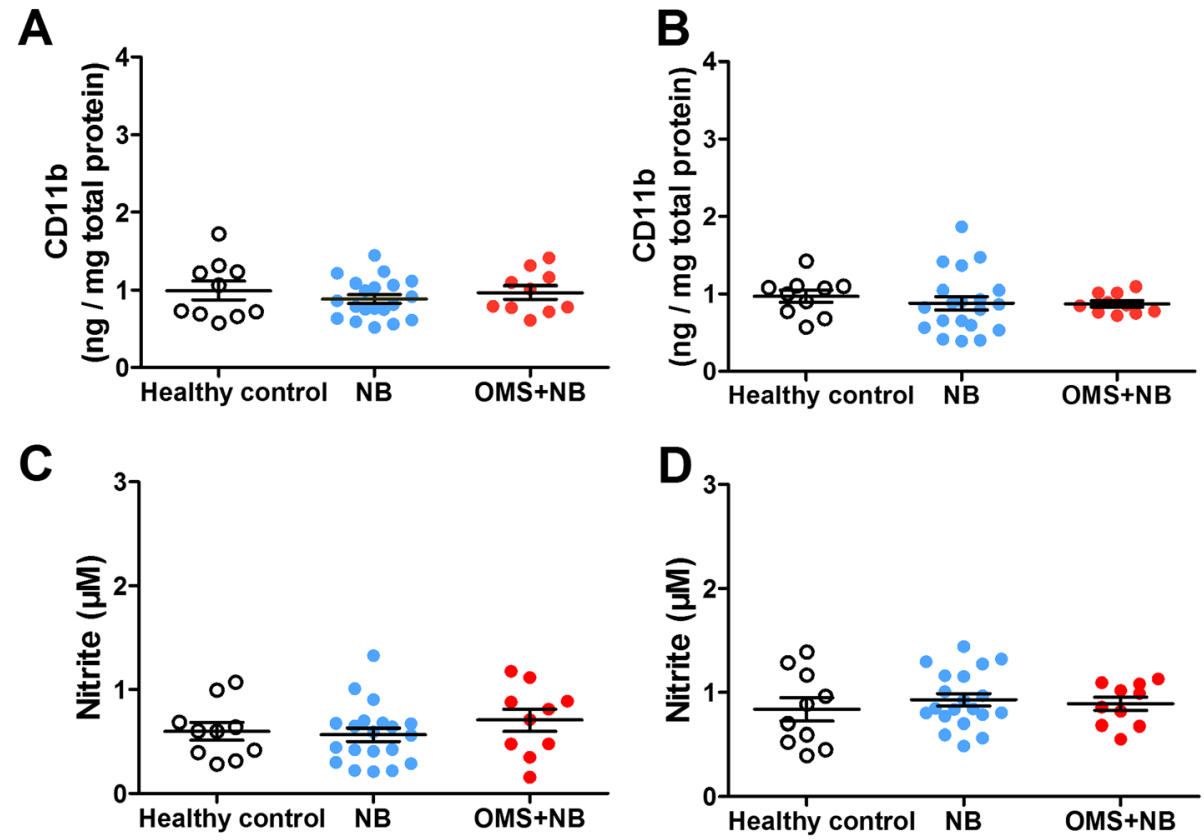

Fig. 10 The Fab fragment of serum IgG cannot induce microglial activation. Note that the expression of CD11b and NO was not significantly changed in cerebral cortical microglias $(\mathbf{a}, \mathbf{b})$ and cerebellar microglias $(\mathbf{c}, \mathbf{d})$ incubated with the Fab fragment of $\lg$ from children with OMS and NB compared with that from NB patients and healthy control. $p>0.05$, one-way ANOVA, $n=10$ (health control), $n=20$ (NB), and $n=10$ (OMS $+\mathrm{NB})$

(Fig. 10c, d). These results suggesting that microglial activation induced by IgG from children with OMS and NB may depend on the Fc fragment of IgG, but not the Fab fragment.

\section{Discussion}

The IgG fraction of sera from children with OMS and NB gives rise to the activation of cultured cerebral cortical and cerebellar microglias

Neuroinflammation in the CNS, a dominant physiological function of microglias and astrocytes, is a common clinical feature in children with OMS [9]. Moreover, the expression of microglial marker soluble CD14 and proinflammatory cytokines is enhanced in CSF from patients with pediatric OMS [10, 11]. Additionally, serum IgG from patients or autoantibody existed in patients directly enhances microglial activation in PD [12], ALS [13], and SLE [14-16] or initially binds with astrocytes or neurons and further indirectly affects microglial activation [42, 43]. Autoantibodies are also detectable in sera and CSF of children with OMS [25, 26] and may be contained in serum IgG from children with OMS and NB in our study, although we did not identify these autoantibodies and autoantibodies include IgM besides IgG. Thus, we hypothesized that serum IgG from children with OMS and NB may impact the activation of microglias. As expected, our results revealed that sera or IgG from children with OMS and NB upregulated the activation of cultured cerebral cortical and cerebellar microglias.

Conversely, commercially available human IgG, or IgG from children with JIA or anti-NMDAR encephalitis, had no such effect, suggesting that the upregulation of microglial activation induced by serum IgG from children with OMS and NB is not simply induced by increased dose of IgG, is not common to all IgG-related diseases, and is not common to all autoantibodymediated disorders of the CNS, indicating specific changes in pediatric OMS at least to some degree. Consistently, we and others found that IgG-induced neuronal cytolysis occurs in pediatric OMS rather than adult OMS [6, 7]. Notably, increased microglial activation is not specific to only OMS, since previous literatures have documented that serum IgG from patients with PD [12] or ALS [13] enhances the activation of microglia and the production of $\mathrm{NO}$, and serum IgG from patients with SLE induced behavioral changes is mediated by microglial activation [14-16]. Moreover, several autoantibodies found in patients with OMS [44-47] also exist in other diseases, such as autoantibody against glycine receptor in progressive encephalomyelitis with rigidity and myoclonus [46, 48] or autoantibody against glutamic acid decarboxylase in stiff-person syndrome [48]. 
Unlike microglial activation, astrocytic activation by serum IgG from children with OMS and NB was not observed by us, indicating that microglia reactivity may be a special mechanism. This notion is supported by studies demonstrating that human immunodeficiency virus infection increases microglial activation but not astrocytic activation [14], and administration of AMD3100 alleviates the pathology of ALS by decreasing microglial activation without affecting astrocytes [17]. However, we cannot completely exclude the role of astrocytes, and the activation of astrocytes may be improved by conditioned media from microglia treated with serum IgG from OMS patients rather than direct stimulation by serum IgG. Furthermore, distinct from the upregulated cytolysis of neurons [6], we observed that the cytolysis of microglias and astrocytes by serum IgG from children with OMS and NB was not changed.

Some cases of pediatric OMS are paraneoplastic and are associated with $\mathrm{NB}$, although varied percentages have been reported [1-5]. The remaining cases are believed to be post-infectious or resulted from NB that has regressed prior to onset of symptoms [48]. Adults with non-paraneoplastic OMS have better outcomes with fewer relapses [47], while children with paraneoplastic and non-paraneoplastic OMS have no significant difference in viral-like prodrome and neurological outcome $[1,49]$. However, whether there is a distinction of paraneoplastic and non-paraneoplastic childhood OMS is still unclear. Our present and previous studies showed that microglial activation or neuronal cytolysis can be induced by serum IgG from children with OMS and NB, but not children with only NB [6], indicating that the IgG fraction may be involved in the pathogenesis of OMS rather than NB. However, we did not obtain sera from children with OMS and without NB; therefore, we cannot completely exclude the possibility that the cytotoxicity induced by serum IgG from children with OMS and NB may be synergistic effects of OMS and NB.

\section{The NO/sGC/PKG pathway takes part in neuronal cytolysis induced by conditioned media from microglias treated with IgG from children with OMS and NB}

A growing body of evidence implicates that microglias in the active state can release various neurotoxic molecules $[8,17]$, causing the loss of neurons in neurodegenerative diseases, including Alzheimer's disease [18], ALS [20], and retinal degeneration [19]. Moreover, we and others have previously demonstrated that sera or IgG from patients with pediatric OMS and NB induces the cytolysis of cerebellar granular and cerebral cortical neurons $[6$, 7]. Consistently, here our results showed that neuronal cytolysis was elevated by conditional media from microglias treated with IgG from children with OMS and NB. Notably, conditioned media from microglias were applied to cultured neurons instead of co-culture of microglias and neurons, and conditioned media were used to cultured neurons after IgG were filtered out, to avoid the direct effects of serum IgG on neurons [6].

$\mathrm{NO}$, synthesized and released by activated microglias, can activate sGC and PKG in neurons, leading to neuronal death by mitochondrial dysfunction [21, 22] and increased neuronal susceptibility to mitochondrial dysfunction [23], which is similar to its function in pancreatic tissues [49]. On the other hand, exposure to NO has anti-apoptotic function through sGC and PKG in both neuronal and myocardial tissues [50, 51], and the opposite influences of $\mathrm{NO}$ intracellular signal may be caused by the way (transient or sustained) and concentration of NO, as well as cell type. In the present study, our results showed the NO/sGC/PKG cascade was a positive regulator of neuronal cytolysis induced by conditioned media from microglias treated with IgG from children with OMS and NB.

Previously, we have documented that IGF-1/PI3K signaling is activated as a compensative mechanism to alleviate neuronal cytolysis directly induced by IgG from sera of children with OMS and NB [6]. Here, we found that the concentration of PI3K was elevated in cerebral cortical and cerebellar neurons incubated with conditioned media from microglias treated with IgG from children with OMS and NB. We also found that exogenous IGF-1 alleviated the cytolysis of neurons incubated with conditioned media, which was attenuated by the PI3K inhibitor (see Additional file 1: Figure S1). These results indicated that IGF-1/PI3K signaling may be compensatively activated to alleviate neuronal lysis induced by IgG through microglias, which is similar to the direct effects of IgG on neuronal lysis. Taken together, IgG from children with OMS and NB increases the activation of microglias, leading to the upregulation of $\mathrm{NO}$, which subsequently activates SGC and PKG in neurons to induce neuronal lysis, at the same time IGF-1/PI3K signaling may be compensatively activated to alleviate neuronal lysis; however, the role of NO and its intracellular cascade seems to be more predominant (Fig. 11).

Interestingly, our results showed that neuronal cytolysis induced by conditioned media from microglias via $\mathrm{NO}$ was almost completely attenuated by the inhibitor of microglia, minocycline. Minocycline has been clinically used for treating infections as an antibiotic, providing a systemic anti-inflammatory effect. Moreover, it produces the anti-inflammatory response in the CNS via microglias and has neuroprotective properties in neurodegenerative diseases, mental illnesses, and others [5256]. In particular, minocycline delays motor alterations, inflammation, and apoptosis in experimental models of PD and ALS [56]. Although there are controversies about its efficacy, the relative safety and tolerability of 


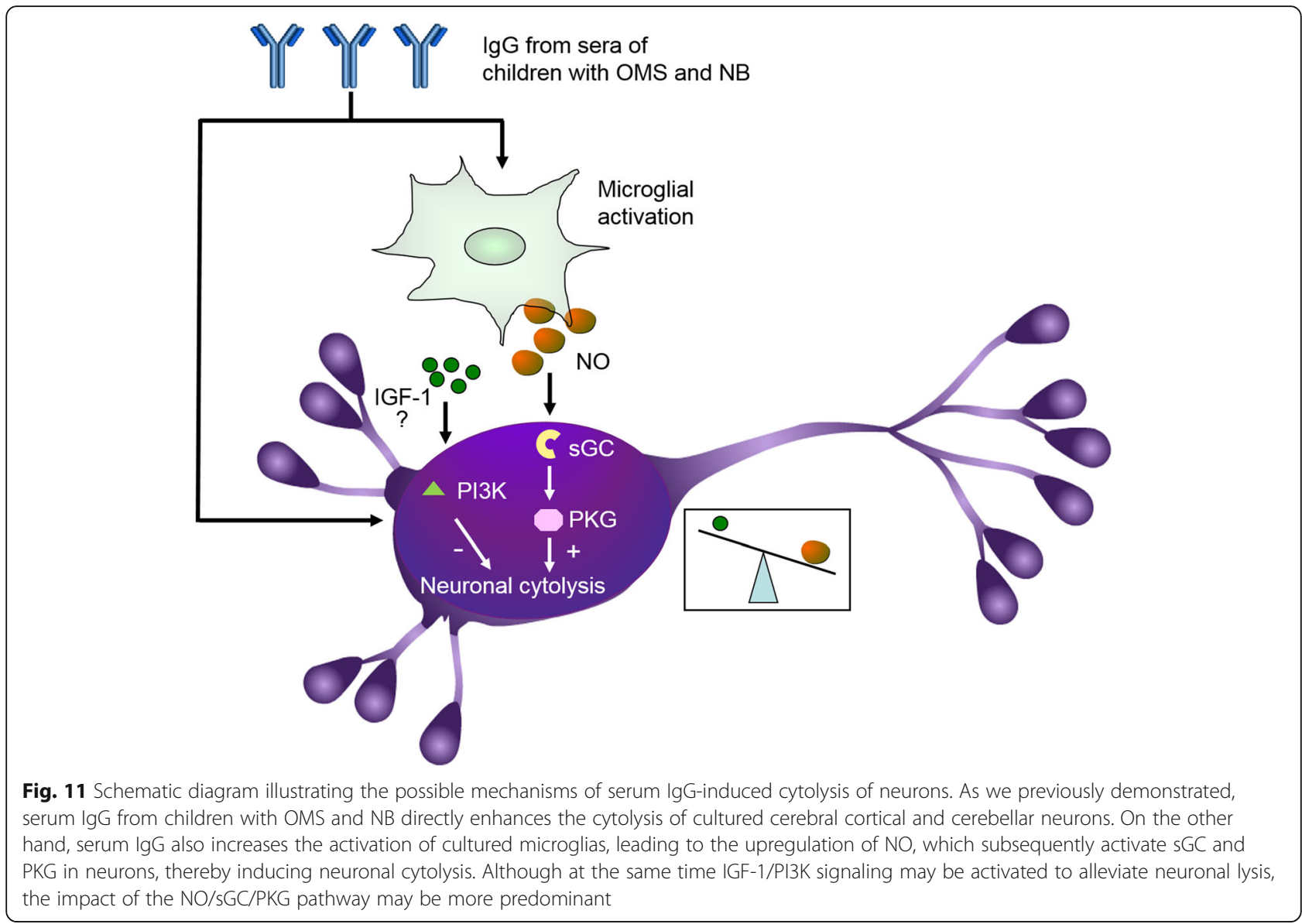

minocycline have led to the launching of various clinical trials; for instance, it may be a possible treatment for patients with acute ischemic stroke [52, 53]. Thus, the administration of minocycline might be an efficient way to treat pediatric OMS.

Although we found that proinflammatory cytokines, namely IL- $1 \beta$, IL- 6 , TNF- $\alpha$, and MCP- 1 , were elevated in the media of microglias treated with IgG from patients with pediatric OMS and NB, and others reported that IL-6 in CSF of untreated OMS patients and IL-1 receptor antagonist in CSF of intravenous immune globulin-treated OMS patients are upregulated [10], the cytolysis of cerebral cortical and cerebellar neurons induced by conditioned media from microglias preincubated with serum IgG was not influenced by these proinflammatory cytokines, indicating a specific effect of the NO pathway on neuronal cytolysis induced by microglial activation. Possible explanations are that cytokines secreted from microglias may take part in other functions rather than neuronal cytolysis, such as the recruitment of immune cells [8], and cytokines enhanced in patients with OMS may be produced by other cells, not microglias.
Microglial activation induced by serum IgG from children with OMS and NB may be through the Fc fragment of IgG $\mathrm{Fc} \gamma \mathrm{R}$, expressed on the surface of microglias, interacts with the Fc fragment of IgG to release proinflammatory cytokines, thereby mediating immune reactions. Several previous studies have documented that IgG combines with $F c \gamma R$ to activate microglias and thus takes part in many diseases. For example, serum IgG from patients with PD significantly induces microglial activation via $\mathrm{F} c \gamma \mathrm{R}$ in the microglia-supplemented neuronal cultures [12]. Also, in an in vitro PD model, neuron-derived IgG activates microglias through Fc $\gamma \mathrm{R}$ [57]. Moreover, autoantibodies can activate microglias through $\mathrm{F} c \gamma \mathrm{R}$ underlie the pathogenesis of autoimmune diseases. For instance, the expression of $\mathrm{Fc} \gamma \mathrm{R}$ crucially contributes to autoantibody-induced tissue injury in experimental epidermolysis bullosa acquisita, an organ-specific autoimmune disease [58]. In agreement with these findings, our results showed that microglial activation may be increased through the Fc fragment of IgG from children with OMS and NB rather than the Fab fragment of IgG. Of interest to note is that we found the activation of microglias was not affected by treatment with 
commercially available human IgG, IgG from children with JIA or anti-NMDAR encephalitis. These findings are consistent with previous reports that IgG combined with microglial Fc $\gamma \mathrm{R}$ can secrete different molecules, either proinflammatory or anti-inflammatory, which depends on the origin and content of $\operatorname{IgG}[12,57]$. Collectively, it is reasonable to speculate that Fc $\gamma R$ may be involved in enhanced microglial activation triggered by the Fc fragment of serum IgG from children with OMS and NB.

\section{Conclusions}

In the present study, we demonstrated that incubation with sera or the IgG fraction from children with OMS and NB upregulates the activation of cultured cerebral cortical and cerebellar microglias. Furthermore, neuronal cytolysis is exerted by incubation with conditioned media from microglias treated with IgG from children with OMS and NB. In addition, the NO/sGC/PKG pathway contributes to neuronal cytolysis induced by conditioned media, and neuronal cytolysis can be almost completely suppressed by pretreatment with the microglial inhibitor minocycline, a clinically tested drug. Finally, increased microglial activation may depend on the Fc fragment of serum IgG rather than the Fab fragment. Our data provide solid evidence that serum IgG from children with OMS and NB increases microglial activation, which induces neuronal cytolysis through the $\mathrm{NO} /$ sGC/PKG pathway, suggesting that the inhibitor of microglia, such as minocycline, may serve as a plausible therapeutic candidate for pediatric OMS.

\section{Supplementary information}

Supplementary information accompanies this paper at https://doi.org/10. 1186/s12974-020-01839-9.

Additional file 1: Figure S1. Effects of IGF-1/PI3K signaling on the cytolysis of neurons induced by conditioned media. The concentration of PI3K was increased in cerebral cortical neurons (a) and cerebellar neurons (b) incubated with conditioned media of the OMS + NB group. The cytolysis of cerebral cortical neurons (c) and cerebellar neurons (d) incubated with conditioned media of the OMS + NB group was alleviated by exogenous IGF-1, which was suppressed by pretreatment with the PI3K inhibitor LY294002. ${ }^{* * *} p<0.001$, one-way ANOVA, $n=20$ (PBS, NB; IGF-1, $\mathrm{NB}), \mathrm{n}=10$ (PBS, OMS+NB; IGF-1 OMS+NB; DMSO, IGF-1, OMS+NB; LY294002, IGF-1, OMS+NB)

\footnotetext{
Abbreviations

7-NINA: 7-Nitroindazole; ALS: Amyotrophic lateral sclerosis; ANOVA: Analysis of variance; BSA: Bovine serum albumin; CD11b: Cluster of differentiation $11 \mathrm{~b}$; CNS: Central nervous system; CSF: Cerebrospinal fluid; DMSO: Dimethyl sulfoxide; ELISA: Enzyme-linked immunosorbent assay; GAPDH: Glyceraldehyde 3-phosphate dehydrogenase; GFAP: Glial fibrillary acidic protein; IGF-1: Insulin-like growth factor 1; IL-1 $\beta$ : Interleukin-1 $\beta$; IL6: Interleukin-6; JIA: Juvenile idiopathic arthritis; LDH: Lactate dehydrogenase; MCP-1: Monocyte chemoattractant protein-1; NB: Neuroblastoma; NMDA: Nmethyl-D-aspartate; NO: Nitric oxide; ODQ: 1H-1,2,4 Oxadiazolo-4,3-a quinoxalin-1-one; OMS: Opsoclonus-myoclonus syndrome; PBS: Phosphate buffer saline; PD: Parkinson disease; PI3K: Phosphoinositide 3-kinase;
}

PKG: Protein kinase G; Po-S: Ponceau S solution; sGC: Soluble guanylyl cyclase; SLE: Systemic lupus erythematosus; SNAP: S-nitroso-Nacetylpenicillamine; TNF-a: Tumor necrosis factor-a; YC-1: 3-5-Hydroxymethyl2-furyl-1-benzyl-indazole

\section{Acknowledgements}

We would like to thank parents and children for their participation in this study.

\section{Authors' contributions}

XD carried out ELISA tests and participated in the design of the study, drafted the manuscript. WY performed the purification of IgG and cytolysis studies. QR carried out primary culture of glial cells and neurons. JH and SY participated in the statistical analysis. JW and XW contributed to the design of the study and the revision of the data. HW conceived the study and participated in the analysis of the data and the draft of the manuscript. All authors read and approved the final manuscript.

\section{Funding}

The present work was supported by grants from the Beiling Talents Fund (2015000021469G204), the Basic Medicine and Clinical Medicine Cooperation Fund of Capital Medical University (15JL70, 16JL20), and the Scientific Research Cultivation Fund of Capital Medical University (PYZ19030).

\section{Availability of data and materials}

The datasets used and/or analyzed during the current study are available from the corresponding author on reasonable request.

\section{Ethics approval and consent to participate}

Ethic approval was granted by the Ethics Committees of Beijing Children's Hospital, Capital Medical University (No. IEC-C-028-A10-V.05). Parents of participants all signed written informed consent. All animals were handled according to the National Institutes of Health Guide for the Care and Use of laboratory animals (NIH Publication No. 8023, revised 1978) in accordance with protocols approved by the Animal Care and Use Committees of Capital Medical University (No. AEEl-2018-034).

\section{Consent for publication}

Not applicable

\section{Competing interests}

The authors declare that they have no competing interests.

\section{Author details}

${ }^{1}$ Laboratory of Nutrition and Development, Beijing Pediatric Research Institute, Beijing Children's Hospital, Capital Medical University, National Center for Children's Health, No. 56 Nan-li-shi Road, Xi-Cheng District, Beijing 100045, China. ${ }^{2}$ Department of Surgical Oncology, Beijing Children's Hospital, Capital Medical University, National Center for Children's Health, Beijing 100045, China. ${ }^{3}$ Department of Neurobiology, School of Basic Medical Sciences, Beijing Institute for Brain Disorders, Capital Medical University, Beijing 100069, China. ${ }^{4}$ Department of Neurology, Beijing Children's Hospital, Capital Medical University, National Center for Children's Health, Beijing 100045, China

Received: 31 December 2019 Accepted: 11 May 2020 Published online: 16 June 2020

\section{References}

1. Krug P, Schleiermacher G, Michon J, Valteau-Couanet D, Brisse H, Peuchmaur $\mathrm{M}$, et al. Opsoclonus-myoclonus in children associated or not with neuroblastoma. Eur J Paediatr Neurol. 2010;14(5):400-9.

2. Pang KK, de Sousa C, Lang B, Pike MG. A prospective study of the presentation and management of dancing eye syndrome/opsoclonusmyoclonus syndrome in the United Kingdom. Eur J Paediatr Neurol. 2010; 14(2):156-61.

3. Hasegawa S, Matsushige T, Kajimoto M, Inoue H, Momonaka H, Oka M, et al. A nationwide survey of opsoclonus-myoclonus syndrome in Japanese children. Brain and Development. 2015;37(7):656-60. 
4. Pranzatelli MR, Tate ED, McGee NR. Demographic, clinical, and immunologic features of 389 children with opsoclonus-myoclonus syndrome: A crosssectional study. Front Neurol. 2017;8:468.

5. Brunklaus A, Pohl K, Zuberi SM, de Sousa C. Investigating neuroblastoma in childhood opsoclonus-myoclonus syndrome. Arch Dis Child. 2012;97(5):461-3.

6. Ding $X$, Han W, Wang J, Yang W, Chang XF, Zhu ZY, et al. IGF-1 alleviates serum lgG-induced neuronal cytolysis through PI3K signaling in children with opsoclonus-myoclonus syndrome and neuroblastoma. Pediatr Res. 2019;85(6):885-94.

7. Fuhlhuber V, Bick S, Tschernatsch M, Dharmalingam B, Kaps M, Preissner KT, et al. Autoantibody-mediated cytotoxicity in paediatric opsoclonusmyoclonus syndrome is dependent on ERK-1/2 phophorylation. J Neuroimmunol. 2015;289:182-6.

8. Subhramanyam CS, Wang C, Hu Q, Dheen ST. Microglia-mediated neuroinflammation in neurodegenerative diseases. Semin Cell Dev Biol. 2019;94:112-20.

9. Pranzatelli MR, McGee NR, Tate ED. Relation of intrathecal oligoclonal band production to inflammatory mediator and immunotherapy response in 208 children with OMS. J Neuroimmunol. 2018;321:150-6.

10. Pranzatelli MR, Tate ED, McGee NR, Colliver JA. Cytokines, cytokine antagonists, and soluble adhesion molecules in pediatric OMS and other neuroinflammatory disorders. J Neurol Sci. 2013;326(1-2):53-8.

11. Pranzatelli MR, Tate ED, McGee NR. Microglial/macrophage markers CHI3L1, sCD14, and SCD163 in CSF and serum of pediatric inflammatory and noninflammatory neurological disorders: A case-control study and reference ranges. J Neurol Sci. 2017;381:285-90.

12. Wang XJ, Yan ZQ, Lu GQ, Stuart S, Chen SD. Parkinson disease IgG and C5ainduced synergistic dopaminergic neurotoxicity: role of microglia. Neurochem Int. 2007;50(1):39-50.

13. Milosevic M, Milicevic K, Bozic I, Lavrnja I, Stevanovic I, Bijelic D, et al. Immunoglobulins $\mathrm{G}$ from sera of amyotrophic lateral sclerosis patients induce oxidative stress and upregulation antioxidative system in BV-2 microglial cell line. Front Immunol. 2017;8:1619.

14. Nestor J, Arinuma Y, Huerta TS, Kowal C, Nasiri E, Kello N, et al. Lupus antibodies induce behavioral changes mediated by microglia and blocked by ACE inhibitors. J Exp Med. 2018;215(10):2554-66.

15. Wang XJ, Li YZ, Wang YC, Feng QH, Yang PT, Qin L. Intracerebroventricula administration of lupus serum induces microglia activation and leukocyte adhesion in the cerebromicrovasculature of mice. J Neuroimmunol. 2019; 334:576994.

16. Yang C, Hou X, Feng Q, Li Y, Wang X, Qin L, Yang P. Lupus serum IgG induces microglia activation through $\mathrm{Fc}$ fragment dependent way and modulated by B-cell activating factor. J Transl Med. 2019;17(1):426.

17. Faissner S, Ambrosius B, Schanzmann K, Grewe B, Potthoff A, Munch J, et al. Cytoplasmic HIV-RNA in monocytes determines microglial activation and neuronal cell death in HIV-associated neurodegeneration. Exp Neurol. 2014; 261:685-97.

18. Biscaro B, Lindvall O, Tesco G, Ekdahl CT, Nitsch RM. Inhibition of microglial activation protects hippocampal neurogenesis and improves cognitive deficits in a transgenic mouse model for Alzheimer's disease. Neurodegener Dis. 2012;9(4):187-98.

19. Scholz R, Sobotka M, Caramoy A, Stempfl T, Moehle C, Langmann T. Minocycline counter-regulates pro-inflammatory microglia responses in the retina and protects from degeneration. J Neuroinflammation. 2015;12:209.

20. Rabinovich-Nikitin I, Ezra A, Barbiro B, Rabinovich-Toidman P, Solomon B. Chronic administration of AMD3100 increases survival and alleviates pathology in SOD1(G93A) mice model of ALS. J Neuroinflammation. 2016;13(1):123.

21. Canals S, Casarejos MJ, de Bernardo S, Rodriguez-Martin E, Mena MA. Nitric oxide triggers the toxicity due to glutathione depletion in midbrain cultures through 12-lipoxygenase. J Biol Chem. 2003;278(24):21542-9.

22. Zhou XY, Zhang F, Ying CJ, Chen J, Chen L, Dong J, et al. Inhibition of iNOS alleviates cognitive deficits and depression in diabetic mice through downregulating the NO/sGC/cGMP/PKG signal pathway. Behav Brain Res. 2017:322:70-82.

23. Mancini A, Tantucci M, Mazzocchetti P, de lure A, Durante $V$, Macchioni $L$, et al. Microglial activation and the nitric oxide/cGMP/PKG pathway underlie enhanced neuronal vulnerability to mitochondrial dysfunction in experimental multiple sclerosis. Neurobiol Dis. 2018;113:97-108.

24. Bataller L, Graus F, Saiz A, Vilchez JJ. Clinical outcome in adult onset idiopathic or paraneoplastic opsoclonus-myoclonus. Brain. 2001;124(Pt 2): 437-43.
25. Blaes F, Pike MG, Lang B. Autoantibodies in childhood opsoclonusmyoclonus syndrome. J Neuroimmunol. 2008;201-202:221-6.

26. Korfei M, Fuhlhuber V, Schmidt-Woll T, Kaps M, Preissner KT, Blaes F. Functional characterisation of autoantibodies from patients with pediatric opsoclonus-myoclonus-syndrome. J Neuroimmunol. 2005;170(1-2):150-7.

27. Yao H, Ma R, Yang L, Hu G, Chen X, Duan M, et al. MiR-9 promotes microglial activation by targeting MCPIP1. Nat Commun. 2014;5:4386.

28. Koistinaho M, Lin SZ, Wu X, Esterman M, Koger D, Hanson J, et al. Apolipoprotein E promotes astrocyte colocalization and degradation of deposited amyloid-beta peptides. Nat Med. 2004;10(7):719-26.

29. Erez Z, Steinberger-Levy I, Shamir M, Doron S, Stokar-Avihail A, Peleg Y, et al. Communication between viruses guides lysis-lysogeny decisions. Nature. 2017;541(7638):488-93.

30. Piotrowska A, Kwiatkowski K, Rojewska E, Makuch W, Mika J. Maraviroc reduces neuropathic pain through polarization of microglia and astroglia Evidence from in vivo and in vitro studies. Neuropharmacology. 2016;108: 207-19.

31. Fernandes NC, Sriram U, Gofman L, Cenna JM, Ramirez SH, Potula R. Methamphetamine alters microglial immune function through P2X7R signaling. J Neuroinflammation. 2016;13(1):91.

32. Bravo J, Lopez-Almaraz R, Mateos M, Diaz L, Hernandez-Exposito S. Neuropsychological profile in opsoclonus-myoclonus-ataxia syndrome presenting as neuroblastic tumours. Rev Neurol. 2016;62(6):249-57.

33. Anand G, Bridge H, Rackstraw P, Chekroud AM, Yong J, Stagg CJ, Pike M. Cerebellar and cortical abnormalities in paediatric opsoclonus-myoclonus syndrome. Dev Med Child Neurol. 2015;57(3):265-72.

34. Kanjanasut N, Phanthumchinda K, Bhidayasiri R. HIV-related opsoclonusmyoclonus-ataxia syndrome: Report on two cases. Clin Neurol Neurosurg. 2010;112(7):572-4.

35. Oh SY, Boegle R, Eulenburg PZ, Ertl M, Kim JS, Dieterich M. Longitudinal multi-modal neuroimaging in opsoclonus-myoclonus syndrome. J Neurol. 2017:264(3):512-9.

36. Panzer JA, Anand R, Dalmau J, Lynch DR. Antibodies to dendritic neuronal surface antigens in opsoclonus myoclonus ataxia syndrome. J Neuroimmunol. 2015;286:86-92.

37. Pifarre P, Garcia A, Mengod G. Species differences in the localization of soluble guanylyl cyclase subunits in monkey and rat brain. J Comp Neurol. 2007:500(5):942-57.

38. Prysyazhna O, Burgoyne JR, Scotcher J, Grover S, Kass D, Eaton P. Phosphodiesterase 5 inhibition limits doxorubicin-induced heart failure by attenuating protein kinase G I oxidation. J Biol Chem. 2016;291(33):1742736.

39. Wu Y, Yuan MM, Su WB, Zhu ML, Yao XY, Wang Y, et al. The constitutively active PKG II mutant effectively inhibits gastric cancer development via a blockade of EGF/EGFR associated signalling cascades. Ther Adv Med Oncol. 2018;10:1758834017751635.

40. Tischkau SA, Mitchell JW, Pace LA, Barnes JW, Barnes JA, Gillette MU. Protein kinase $\mathrm{G}$ type $\mathrm{Il}$ is required for night-to-day progression of the mammalian circadian clock. Neuron. 2004;43(4):539-49.

41. Wu CL, Chen SD, Yin JH, Hwang CS, Yang DI. Nuclear factor-kappaBdependent sestrin2 induction nediates the antioxidant effects of BDNF against mitochondrial inhibition in rat cortical neurons. Mol Neurobiol. 2016; 53(6):4126-42

42. Giannoccaro MP, Menassa DA, Jacobson L, Coutinho E, Prota G, Lang B, et al. Behaviour and neuropathology in mice injected with human contactinassociated protein 2 antibodies. Brain. 2019;142(7):2000-12.

43. Yick LW, Ma OKF, Ng RCL, Kwan JSC, Chanh KH. Aquaporin-4 autoantibodies from neuromyelitis optica spectrum disorder patients induce complementindependent immunopathologies in mice. Front Immunol. 2018;9:1438.

44. Lenti C, Bognetti E, Bonfanti R, Bonifacio E, Meschi F. Myoclonic Encephalopathy and Diabetes Mellitus in a Boy. Dev Med Child Neurol. 1999;41(7):489-90.

45. Markakis I, Alexiou E, Xifaras M, Gekas G, Rombos A. Opsoclonus-myoclonusataxia syndrome with autoantibodies to glutamic acid decarboxylase. Clin Neurol Neurosurg. 2008;110(6):619-21.

46. lizuka T, Leite MI, Lang B, Waters P, Urano Y, Miyakawa S, et al. Glycine receptor antibodies are detected in progressive encephalomyelitis with rigidity and myoclonus (PERM) but not in saccadic oscillations. J Neurol. 2012;259(8):1566-73.

47. Armangue $T$, Sabater $L$, Torres-Vega E, Martinez-Hernandez E, Arino H, PetitPedrol $M$, et al. Clinical and immunological features of opsoclonus- 
myoclonus syndrome in the era of neuronal cell surface Antibodies. JAMA Neurol. 2016;73(4):417-24.

48. Alexopoulos $\mathrm{H}$, Dalakas MC. The immunobiology of autoimmune encephalitides. J Autoimmun. 2019:104:102339.

49. Tejedo J, Bernabe JC, Ramirez R, Sobrino F, Bedoya FJ. NO induces a cGMPindependent release of cytochrome $\mathrm{c}$ from mitochondria which precedes caspase 3 activation in insulin producing RINm5F cells. FEBS Lett. 1999; 459(2):238-43.

50. Chen SD, Wu CL, Hwang WC, Yang DI. More insight into BDNF against neurodegeneration: anti-apoptosis, anti-oxidation, and suppression of autophagy. Int J Mol Sci. 2017;18(3).

51. Arandarcikaite $\mathrm{O}$, Jokubka R, Borutaite $\mathrm{V}$. Neuroprotective effects of nitric oxide donor NOC-18 against brain ischemia-induced mitochondrial damages: role of PKG and PKC. Neurosci Lett. 2015;586:65-70.

52. Sutherland BA, Minnerup J, Balami JS, Arba F, Buchan AM, Kleinschnitz C, et al. Neuroprotection for ischaemic stroke: translation from the bench to the bedside. Int J Stroke. 2012;7(5):407-18.

53. Malhotra K, Chang JJ, Khunger A, Blacker D, Switzer JA, Goyal N, et al. Minocycline for acute stroke treatment: a systematic review and metaanalysis of randomized clinical trials. J Neurol. 2018;265(8):1871-9.

54. Zhang $L$, Zheng $H$, Wu R, Kosten TR, Zhang XY, Zhao J. The effect of minocycline on amelioration of cognitive deficits and pro-inflammatory cytokines levels in patients with schizophrenia. Schizophr Res. 2019;212:928.

55. Amani M, Shokouhi G, Salari AA. Minocycline prevents the development of depression-like behavior and hippocampal inflammation in a rat model of Alzheimer's disease. Psychopharmacology. 2019;236(4):1281-92.

56. Blum D, Chtarto A, Tenenbaum L, Brotchi J, Levivier M. Clinical potential of minocycline for neurodegenerative disorders. Neurobiol Dis. 2004;17(3):35966.

57. Zhang J, Niu N, Wang M, McNutt MA, Zhang D, Zhang B, et al. Neuronderived lgG protects dopaminergic neurons from insult by 6-OHDA and activates microglia through the FcgammaR I and TLR4 pathways. Int J Biochem Cell Biol. 2013;45(8):1911-20.

58. Kasperkiewicz M, Nimmerjahn F, Wende S, Hirose M, Iwata H, Jonkman MF, et al. Genetic identification and functional validation of FcyRIV as key molecule in autoantibody-induced tissue injury. J Pathol. 2012;228(1):8-19.

\section{Publisher's Note}

Springer Nature remains neutral with regard to jurisdictional claims in published maps and institutional affiliations.

Ready to submit your research? Choose BMC and benefit from:

- fast, convenient online submission

- thorough peer review by experienced researchers in your field

- rapid publication on acceptance

- support for research data, including large and complex data types

- gold Open Access which fosters wider collaboration and increased citations

- maximum visibility for your research: over $100 \mathrm{M}$ website views per year

At $\mathrm{BMC}$, research is always in progress.

Learn more biomedcentral.com/submissions 